\title{
BBGKY chain of kinetic equations, non-equilibrium statistical operator method and collective variable method in the statistical theory of non-equilibrium liquids
}

\author{
I.R. Yukhnovskii, P.A. Hlushak, M.V. Tokarchuk \\ Institute for Condensed Matter Physics of the National Academy of Sciences of Ukraine, \\ 1 Svientsitskii St., 79011 Lviv, Ukraine
}

Received July 6, 2016, in final form November 1, 2016

\begin{abstract}
A chain of kinetic equations for non-equilibrium one-particle, two-particle and $s$-particle distribution functions of particles which take into account nonlinear hydrodynamic fluctuations is proposed. The method of Zubarev non-equilibrium statistical operator with projection is used. Nonlinear hydrodynamic fluctuations are described with non-equilibrium distribution function of collective variables that satisfies generalized Fokker-Planck equation. On the basis of the method of collective variables, a scheme of calculation of non-equilibrium structural distribution function of collective variables and their hydrodynamic speeds (above Gaussian approximation) contained in the generalized Fokker-Planck equation for the non-equilibrium distribution function of collective variables is proposed. Contributions of short- and long-range interactions between particles are separated, so that the short-range interactions (for example, the model of hard spheres) are described in the coordinate space, while the long-range interactions - in the space of collective variables. Short-ranged component is regarded as basic, and corresponds to the BBGKY chain of equations for the model of hard spheres.
\end{abstract}

Key words: non-linear fluctuations, non-equilibrium statistical operator, distribution function, Fokker-Planck equation, simple fluid

PACS: $74.40 . G h, 05.70 . L, 64.70 . F$

\section{Introduction}

A development of equilibrium and non-equilibrium statistical mechanics of classical and quantum systems, which began in 1970-ies following the work by Bogoliubov [1] and works by Born, Green [2], Kirkwood [3, 4], Yvon [5] and continues today, has led to a substantial progress in the theory of gases, liquids, plasma. Bogoliubov in the book [1], which turns 70 years, in a strict form formulated the idea of a hierarchy of relaxation times in a system of many interacting particles and a reduced number of parameters describing the evolution of the system. This idea played a major role in the development of modern methods of non-equilibrium theory to study the dynamics of macroscopic systems at kinetic and hydrodynamic stages, based on the fundamental principles of statistical mechanics.

Important for the development of this direction were the works by Zubarev [6-8], Zwanzig [9-11], Robertson [12, 13], Kawasaki and Gunton [14], Peletminskii and Yatsenko [15], Zubarev and Kalashnikov [16]. The results of the research done in this field are detailed in books [17-23] and reviews [24, 25]. However, along with the important results of theories in statistical physics, as well as in other fields of modern physics, there are still many unsolved problems, especially in the theory of non-equilibrium processes.

In dense gases and fluids in the field of phase transitions, the heterophase fluctuations play an important role [26-34]. They always appear and disappear during the diffusion processes. In the field of 
phase transitions, the heterophase fluctuations are factors forming a new phase, in particular, forming the bubbles in a liquid or drops in a gas.

The non-equilibrium gas-liquid phase transition is characterized by nonlinear hydrodynamic fluctuations of mass, momentum and particle energy, which describe a collective nature of the process and define the spatial and temporal behavior of the transport coefficients (viscosity, thermal conductivity), time correlation functions and a dynamic structure factor. At the same time, due to heterogeneity in collective dynamics of these fluctuations, liquid drops emerge in the gas phase (in case of transition from the gas phase to the liquid phase), or the gas bubbles emerge in the liquid phase (in case of transition from the liquid phase to the gas phase), the formation of which is of a kinetic nature described by a redistribution of momentum and energy, i.e., when a certain group of particles in the system receives a significant decrease (in the case of drops), or increase (in the case of bubbles) of kinetic energy. The particles, that form bubbles or droplets, diffuse out of their phases in the liquid or in the gas and vice versa. They have different values of momentum, energy and pressure in different phases. All these features are related to the non-equilibrium one-, two-, s-particle distribution functions (which depend on the coordinate, momentum and time) that satisfy the Bogoliubov-Born-Green-Kirkwood-Yvon (BBGKY) chain of equations. They are the real heterophase systems in which bubble embryoses, drops or small crystals are of a kinetic nature caused by nonlinear fluctuations, changes in temperature, pressure. In our case, the heterophase formations (containing a finite particle number in this or that phase) can be described by non-equilibrium distribution function $f_{s}\left(x^{s} ; t\right)$. The kinetic processes within heterophase formations are described respectively by the kinetic equations [35], in which the right-hand side contains the summands that take into account the mutual effect of kinetic and hydrodynamic processes. Obviously, such heterophase formations form and disappear (with finite lifetime), exchanging by both the particles and energy with the surrounding particles in the background of nonlinear hydrodynamic fluctuations of densities of particle number, momentum, energy; the contribution of such fluctuations grows at phase transformations. These nonlinear fluctuations are described by the Fokker-Planck equation [35]. Here, in the processes of interaction between kinetic and hydrodynamic fluctuations with appropriate changes of temperature and pressure in heterophase formations due to spontaneous symmetry breaking there can be self-organizing processes of sorts of particle motion with group velocity $f_{s}\left(x^{s} ; t\right)=f_{s}\left(\mathbf{r}_{1}-\mathbf{v} t, \mathbf{p}_{1}, \ldots, \mathbf{r}_{s}-\mathbf{v} t, \mathbf{p}_{s}\right)$, which leads to an automodel (quasi-soliton) spreading of heterophase formations in the respective system.

Such processes require a detailed separate study due to the calculation difficulties of kinetic and hydrodynamic transport kernels in equations of transport. In this connection we would like to draw attention to the Klimontovich article [36], in which to a certain extent there is realized a consistent description of kinetics and hydrodynamics (diffusion processes are taken into account) for gas-liquid phase transition.

The main difficulty is that the kinetics and hydrodynamics of these processes are strongly interrelated and should be considered simultaneously. In articles [37-39] there is proposed a consistent description of kinetic and hydrodynamic processes in dense gases and liquids on the basis of Zubarev non-equilibrium statistical operator [19, 20].

This article is an extension or generalization of article [35]. Here, we want to emphasize certain aspects. In [35], the main parameter for the description of kinetic processes was one-particle non-equilibrium distribution function that corresponds to the gas description. In order to get a consistent description of kinetic and hydrodynamic processes (which is important for dense gases and liquids [37-39]) it is necessary to include the density of average potential energy as an important parameter. Having included this parameter, we can identify explicit contributions connected with short- and long-range interactions between particles. For dilute gases, this parameter can be neglected, while for dense gases and liquids one makes an important contribution compared to kinetic energy. In this contribution, we will develop an approach [35] for a consistent description of kinetic and hydrodynamic processes that are characterized by non-linear fluctuations taking into account short-range and long-range interactions between particles. The approach is important for the description of non-equilibrium gas-liquid phase transition.

In Section 2 we will obtain the non-equilibrium statistical operator for non-equilibrium state of the system when the parameters of a reduced description are represented by a non-equilibrium one-particle distribution function, the density of non-equilibrium average value of potential energy of particle interaction and a distribution function of non-equilibrium nonlinear hydrodynamic variables. Using this operator, we construct kinetic equations for non-equilibrium one-, two-, s-particle distribution functions 
which take into account nonlinear hydrodynamic fluctuations, for which the non-equilibrium distribution function satisfies a generalized Fokker-Planck equation.

In Section 3 we will consider a scheme for calculation of the structural distribution function of hydrodynamic collective variables and their hydrodynamic velocities (in approximation higher than Gaussian), which enter the generalized Fokker-Planck equation for the non-equilibrium distribution function of hydrodynamic collective variables. We separate the contributions from short-range and long-range interactions between particles, which will be described in the coordinate space and in the space of collective variables, respectively. Moreover, the short-range component will be considered in a simplified manner, which in our case will correspond to the BBGKY chain of equations for the model of hard spheres [40].

\section{Non-equilibrium distribution function and BBGKY chain of kinetic eq- uations in the Zubarev non-equilibrium statistical operator method}

For a consistent description of kinetic and hydrodynamic fluctuations in a classical one-component fluid, it is necessary to select the description parameters for one-particle and collective processes. For these parameters, we choose the non-equilibrium one-particle distribution function $f_{1}(x ; t)=\left\langle\hat{n}_{1}(x)\right\rangle^{t}$, the density of non-equilibrium average value of potential energy of particle interaction $H^{\text {int }}(\mathbf{r}, t)=$ $\left\langle\hat{H}^{\mathrm{int}}(\mathbf{r})\right\rangle^{t}$ and distribution function of hydrodynamic variables $f(a ; t)=\langle\delta(\hat{a}-a)\rangle^{t}$. Here, the phase function

$$
\hat{n}_{1}(x)=\sum_{j=1}^{N} \delta\left(x-x_{j}\right)=\sum_{j=1}^{N} \delta\left(\mathbf{r}-\mathbf{r}_{j}\right) \delta\left(\mathbf{p}-\mathbf{p}_{j}\right)
$$

is the microscopic particle number density. $x_{j}=\left(\mathbf{r}_{j}, \mathbf{p}_{j}\right)$ is the set of phase variables (coordinates and momenta), $N$ is the total number of particles in a volume $V$.

$$
\hat{H}^{\mathrm{int}}(\mathbf{r})=\frac{1}{2} \sum_{j \neq l=1}^{N} \Phi\left(\left|\mathbf{r}_{l j}\right|\right) \delta\left(\mathbf{r}-\mathbf{r}_{j}\right)
$$

is the microscopic density of the potential energy of particle interaction. A microscopic phase distribution of hydrodynamic variables is given by

$$
\hat{f}(a)=\delta(\hat{a}-a)=\prod_{l=1}^{3} \prod_{\mathbf{k}} \delta\left(\hat{a}_{l \mathbf{k}}-a_{l \mathbf{k}}\right)
$$

where $\hat{a}_{1 \mathbf{k}}=\hat{n}_{\mathbf{k}}, \hat{a}_{2 \mathbf{k}}=\hat{\mathbf{J}}_{\mathbf{k}}, \hat{a}_{3 \mathbf{k}}=\hat{\varepsilon}_{\mathbf{k}}$ are the Fourier components of the densities of particle number, momentum and energy:

$$
\hat{n}_{\mathbf{k}}=\sum_{j=1}^{N} \mathrm{e}^{-\mathrm{i} \mathbf{k r}_{j}}, \quad \hat{\mathbf{J}}_{\mathbf{k}}=\sum_{j=1}^{N} \mathbf{p}_{j} \mathrm{e}^{-\mathrm{i} \mathbf{k} \mathbf{r}_{j}}, \quad \hat{\varepsilon}_{\mathbf{k}}=\sum_{j=1}^{N}\left[\frac{p_{j}^{2}}{2 m}+\frac{1}{2} \sum_{l \neq j=1}^{N} \Phi\left(\left|\mathbf{r}_{l j}\right|\right)\right] \mathrm{e}^{-\mathrm{i} \mathbf{k} \mathbf{r}_{j}},
$$

and $a_{m \mathbf{k}}=\left(n_{\mathbf{k}}, \mathbf{J}_{\mathbf{k}}, \varepsilon_{\mathbf{k}}\right)$ are the corresponding collective variables. $\Phi\left(\left|\mathbf{r}_{l j}\right|\right)=\Phi\left(\left|\mathbf{r}_{l}-\mathbf{r}_{j}\right|\right)$ is the pair interaction potential between particles, which we assume can be represented as the sum of short-range interaction $\Phi^{\mathrm{sh}}\left(\left|\mathbf{r}_{l j}\right|\right)$ and long-range interaction $\Phi^{\text {long }}\left(\left|\mathbf{r}_{l j}\right|\right)$ potentials:

$$
\Phi\left(\left|\mathbf{r}_{l j}\right|\right)=\Phi^{\mathrm{sh}}\left(\left|\mathbf{r}_{l j}\right|\right)+\Phi^{\text {long }}\left(\left|\mathbf{r}_{l j}\right|\right) .
$$

The introduction of $\left\langle\hat{H}^{\mathrm{int}}(\mathbf{r})\right\rangle^{t}$ as a parameter is important step, as for liquids and dense gases it gives a larger contribution than the kinetic part of energy connected with $\left\langle\hat{n}_{1}(x)\right\rangle^{t}$. In addition this parameter describes the collective dynamics of short- and long-range interactions:

$$
\left\langle\hat{H}^{\mathrm{int}}(\mathbf{r})\right\rangle^{t}=\left\langle\hat{H}^{\mathrm{sh}}(\mathbf{r})\right\rangle^{t}+\frac{1}{2 V^{2}} \sum_{\mathbf{q}, \mathbf{k}} v(\mathbf{k}) \mathrm{e}^{\mathrm{iqr}}\left(\left\langle\hat{n}_{\mathbf{q}+\mathbf{k}} \hat{n}_{-\mathbf{k}}\right\rangle^{t}-\left\langle\hat{n}_{\mathbf{q}}\right\rangle^{t}\right),
$$

$\left\langle\hat{n}_{\mathbf{q}+\mathbf{k}} \hat{n}_{-\mathbf{k}}\right\rangle^{t}=F(\mathbf{q}, \mathbf{k} ; t)$ is the non-equilibrium scattering function which connected with non-equilibrium dynamic factor of system, $v(\mathbf{k})=\int \mathrm{d} \mathbf{r} \mathrm{e}^{-\mathrm{ikr}} \Phi^{\text {long }}(|\mathbf{r}|)$ is the Fourier component of long-range potential of 
particle interactions. The average values $\left\langle\hat{n}_{1}(x)\right\rangle^{t},\left\langle\hat{H}^{\text {int }}(\mathbf{r})\right\rangle^{t}$ and $\langle\delta(\hat{a}-a)\rangle^{t}$ are calculated by means of the non-equilibrium $N$-particle distribution function $\varrho\left(x^{N} ; t\right)$, that satisfies the Liouville equation. In line with the idea of reduced description of non-equilibrium states this function is the functional

$$
\varrho\left(x^{N} ; t\right)=\varrho\left(\ldots, f_{1}(x ; t),\left\langle\hat{H}^{\text {int }}(\mathbf{r})\right\rangle^{t}, f(a ; t), \ldots\right) .
$$

In order to find a non-equilibrium distribution function $\varrho\left(x^{N} ; t\right)$ we use Zubarev's method [19, 20, 41], in which a general solution of Liouville equation taking into account a projection procedure can be presented in the form:

$$
\varrho\left(x^{N} ; t\right)=\varrho_{\text {rel }}\left(x^{N} ; t\right)-\int_{-\infty}^{t} \mathrm{~d} t^{\prime} \mathrm{e}^{\epsilon\left(t^{\prime}-t\right)} T\left(t, t^{\prime}\right)\left[1-P_{\text {rel }}\left(t^{\prime}\right)\right] \mathrm{i} L_{N} \varrho_{\text {rel }}\left(x^{N} ; t^{\prime}\right),
$$

where $\epsilon \rightarrow+0$ after thermodynamic limiting transition. The source selects the retarded solutions of Liouville equation with operator $\mathrm{i} L_{N} . T\left(t, t^{\prime}\right)=\exp _{+}\left\{-\int_{t^{\prime}}^{t} \mathrm{~d} t^{\prime}\left[1-P_{\text {rel }}\left(t^{\prime}\right)\right] \mathrm{i} L_{N}\right\}$ is the generalized time evolution operator taking into account Kawasaki-Gunton projection $P_{\text {rel }}\left(t^{\prime}\right)$. The structure of $P_{\text {rel }}\left(t^{\prime}\right)$ depends on the relevant distribution function $\varrho_{\text {rel }}\left(x^{N} ; t\right)$, which in method by Zubarev is determined from extremum of the information entropy at simultaneous conservation of normalization condition

$$
\int \mathrm{d} \Gamma_{N} \varrho_{\text {rel }}\left(x^{N} ; t\right)=1, \quad \mathrm{~d} \Gamma_{N}=\frac{(\mathrm{d} x)^{N}}{\mathrm{~h}^{3 N} N !}=\frac{\left(\mathrm{d} x_{1}, \ldots, \mathrm{d} x_{N}\right)}{\mathrm{h}^{3 N} N !}, \quad \mathrm{d} x=\mathrm{d} \mathbf{r d} \mathbf{p},
$$

and the fact that the parameters of the reduced description, $f_{1}(x ; t)$ and $f(a ; t)$ are fixed. Then, a relevant distribution function can be written as follows:

$$
\varrho_{\text {rel }}\left(x^{N} ; t\right)=\exp \left[-\Phi(t)-\int \mathrm{d} x \gamma(x ; t) \hat{n}_{1}(x)-\int \mathrm{d} \mathbf{r} \beta(\mathbf{r} ; t) \hat{H}^{\mathrm{int}}(\mathbf{r})-\int \mathrm{d} a F(a ; t) \hat{f}(a)\right],
$$

where $\mathrm{d} a$ is the integration over collective variables:

$$
\begin{array}{r}
\mathrm{d} a=\prod_{\mathbf{k}} \mathrm{d} n_{\mathbf{k}} \mathrm{d} \mathbf{j}_{\mathbf{k}} \mathrm{d} \varepsilon_{\mathbf{k}}, \quad \mathrm{d} n_{\mathbf{k}}=\mathrm{d} \operatorname{Re} n_{\mathbf{k}} \mathrm{d} \operatorname{Im} n_{\mathbf{k}}, \quad \mathrm{d} \varepsilon_{\mathbf{k}}=\mathrm{d} \operatorname{Re} \varepsilon_{\mathbf{k}} \mathrm{d} \operatorname{Im} \varepsilon_{\mathbf{k}}, \\
\mathrm{d} \mathbf{j}_{\mathbf{k}}=\mathrm{d} j_{x, \mathbf{k}} \mathrm{d} j_{y, \mathbf{k}} \mathrm{d} j_{z, \mathbf{k}}, \quad \mathrm{d} j_{x, \mathbf{k}}=\mathrm{d} \operatorname{Re} j_{x, \mathbf{k}} \mathrm{d} \operatorname{Im} j_{x, \mathbf{k}}, \ldots
\end{array}
$$

The Massieu-Planck functional $\Phi(t)$ is determined from the normalization condition for the relevant distribution function

$$
\Phi(t)=\ln \int \mathrm{d} \Gamma_{N} \exp \left[-\int \mathrm{d} x \gamma(x ; t) \hat{n}_{1}(x)-\int \mathrm{d} \mathbf{r} \beta(\mathbf{r} ; t) \hat{H}^{\mathrm{int}}(\mathbf{r})-\int \mathrm{d} a F(a ; t) \hat{f}(a)\right] .
$$

The functions $\gamma(x ; t), \beta(\mathbf{r} ; t)$ and $F(a ; t)$ are the Lagrange multipliers and are determined from the self-consistent conditions:

$$
\begin{gathered}
f_{1}(x ; t)=\left\langle\hat{n}_{1}(x)\right\rangle^{t}=\left\langle\hat{n}_{1}(x)\right\rangle_{\text {rel }}^{t},\left\langle\hat{H}^{\text {int }}(\mathbf{r})\right\rangle^{t}=\left\langle\hat{H}^{\text {int }}(\mathbf{r})\right\rangle_{\text {rel }}^{t}, \\
f(a ; t)=\langle\delta(\hat{a}-a)\rangle^{t}=\langle\delta(\hat{a}-a)\rangle_{\text {rel }}^{t},
\end{gathered}
$$

where $\langle\ldots\rangle^{t}=\int \mathrm{d} \Gamma_{N} \ldots \varrho\left(x^{N} ; t\right)$ and $\langle\ldots\rangle_{\text {rel }}^{t}=\int \mathrm{d} \Gamma_{N} \ldots \varrho_{\text {rel }}\left(x^{N} ; t\right)$. To find the explicit form of non-equilibrium distribution function $\varrho\left(x^{N} ; t\right)$, we exclude the factor $F(a ; t)$ in a relevant distribution function and thereafter, by means of self-consistent conditions (2.9), we have

$$
\varrho_{\text {rel }}\left(x^{N} ; t\right)=\left.\varrho_{\text {rel }}^{\text {kin-hyd }}\left(x^{N} ; t\right) \frac{f(a ; t)}{W(a ; t)}\right|_{a=\hat{a}} .
$$

Here,

$$
\begin{aligned}
W(a ; t) & =\int \mathrm{d} \Gamma_{N} \exp \left[-\Phi^{\text {kin-hyd }}(t)-\int \mathrm{d} x \gamma(x ; t) \hat{n}_{1}(x)-\int \mathrm{d} \mathbf{r} \beta(\mathbf{r} ; t) \hat{H}^{\mathrm{int}}(\mathbf{r})\right] \hat{f}(a) \\
& =\int \mathrm{d} \Gamma_{N} \varrho_{\mathrm{rel}}^{\text {kin-hyd }}\left(x^{N} ; t\right) \hat{f}(a)
\end{aligned}
$$


is the structure distribution function of hydrodynamic variables, which could be also considered as a Jacobian for transition from $\hat{f}(a)$ into space of collective variables $n_{\mathbf{k}}, \mathbf{J}_{\mathbf{k}}, \varepsilon_{\mathbf{k}}$ averaged with the "kinetic" relevant distribution function

$$
\begin{aligned}
\varrho_{\text {rel }}^{\text {kin-hyd }}\left(x^{N} ; t\right) & =\exp \left[-\Phi^{\text {kin-hyd }}(t)-\int \mathrm{d} x \gamma(x ; t) \hat{n}_{1}(x)-\int \mathrm{d} \mathbf{r} \beta(\mathbf{r} ; t) \hat{H}^{\mathrm{int}}(\mathbf{r})\right], \\
\Phi^{\text {kin-hyd }}(t) & =\ln \int \mathrm{d} \Gamma_{N} \exp \left[-\int \mathrm{d} x \gamma(x ; t) \hat{n}_{1}(x)-\int \mathrm{d} \mathbf{r} \beta(\mathbf{r} ; t) \hat{H}^{\mathrm{int}}(\mathbf{r})\right] .
\end{aligned}
$$

Here, the entropy

$$
\begin{aligned}
S(t)=-\left\langle\ln \varrho_{\text {rel }}\left(x^{N} ; t\right)\right\rangle_{\text {rel }}^{t}= & \Phi(t)+\int \mathrm{d} x \gamma(x ; t)\left\langle\hat{n}_{1}(x)\right\rangle^{t}+\int \mathrm{d} \mathbf{r} \beta(\mathbf{r} ; t)\left\langle\hat{H}^{\mathrm{int}}(\mathbf{r})\right\rangle^{t} \\
& +\int \mathrm{d} a f(a ; t) \ln \frac{f(a ; t)}{W(a ; t)} .
\end{aligned}
$$

corresponds to the relevant distribution (2.10). In combination with the self-consistent conditions [2.9), it can be considered as entropy of non-equilibrium state. In accordance with (2.6), in order to obtain the explicit form of non-equilibrium distribution function, it is necessary to disclose the action of Liouville operator on $\varrho_{\text {rel }}\left(x^{N} ; t\right)$ and the action of the Kawasaki-Gunton projection operator, which in our case has the following structure according to 2.10 :

$$
\begin{aligned}
P_{\text {rel }}(t) \varrho^{\prime}= & \varrho_{\text {rel }}\left(x^{N} ; t\right) \int \mathrm{d} \Gamma_{N} \varrho^{\prime}+\int \mathrm{d} x \frac{\partial \varrho_{\text {rel }}\left(x^{N} ; t\right)}{\partial\left\langle\hat{n}_{1}(x)\right\rangle^{t}}\left[\int \mathrm{d} \Gamma_{N} \hat{n}_{1}(x) \varrho^{\prime}-\left\langle\hat{n}_{1}(x)\right\rangle^{t} \int \mathrm{d} \Gamma_{N} \varrho^{\prime}\right] \\
& +\int \mathrm{d} \mathbf{r} \frac{\partial \varrho_{\operatorname{rel}}\left(x^{N} ; t\right)}{\partial\left\langle\hat{H}^{\mathrm{int}}(\mathbf{r})\right\rangle^{t}}\left[\int \mathrm{d} \Gamma_{N} \hat{H}^{\mathrm{int}}(\mathbf{r}) \varrho^{\prime}-\left\langle\hat{H}^{\mathrm{int}}(\mathbf{r})\right\rangle^{t} \int \mathrm{d} \Gamma_{N} \varrho^{\prime}\right] \\
& +\int \mathrm{d} a \frac{\partial \varrho_{\mathrm{rel}}\left(x^{N} ; t\right)}{\partial\left[\frac{f(a ; t)}{W(a ; t)}\right]} \frac{1}{W(a ; t)}\left[\int \mathrm{d} \Gamma_{N} \hat{f}(a) \varrho^{\prime}-f(a ; t) \int \mathrm{d} \Gamma_{N} \varrho^{\prime}\right] \\
& +\int \mathrm{d} x \int \mathrm{d} a \frac{\partial \varrho_{\text {rel }}\left(x^{N} ; t\right)}{\partial\left[\frac{f(a ; t)}{W(a ; t)}\right]} \frac{f(a ; t)}{W(a ; t)} \frac{\partial \ln W(a ; t)}{\partial\left\langle\hat{n}_{1}(x)\right\rangle^{t}}\left[\int \mathrm{d} \Gamma_{N} \hat{n}_{1}(x) \varrho^{\prime}-\left\langle\hat{n}_{1}(x)\right\rangle^{t} \int \mathrm{d} \Gamma_{N} \varrho^{\prime}\right] \\
& +\int \mathrm{d} \mathbf{r} \int \mathrm{d} a \frac{\partial \varrho_{\text {rel }}\left(x^{N} ; t\right)}{\partial\left[\frac{f(a ; t)}{W(a ; t)}\right]} \frac{f(a ; t)}{W(a ; t)} \frac{\partial \ln W(a ; t)}{\partial\left\langle\hat{H}^{\mathrm{int}}(\mathbf{r})\right\rangle^{t}}\left[\int \mathrm{d} \Gamma_{N} \hat{H}^{\text {int }}(\mathbf{r}) \varrho^{\prime}-\left\langle\hat{H}^{\text {int }}(\mathbf{r})\right\rangle^{t} \int \mathrm{d} \Gamma_{N} \varrho^{\prime}\right] .
\end{aligned}
$$

Next, we consider the action of Liouville operator on a relevant distribution function [2.10):

$$
\begin{aligned}
\mathrm{i} L_{N} \varrho_{\text {rel }}\left(x^{N} ; t\right)= & -\int \mathrm{d} x \gamma(x ; t) \dot{\hat{n}}_{1}(x) \varrho_{\text {rel }}\left(x^{N} ; t\right)-\int \mathrm{d} \mathbf{r} \beta(\mathbf{r} ; t) \dot{\hat{H}}^{\text {int }}(\mathbf{r}) \varrho_{\text {rel }}\left(x^{N} ; t\right) \\
& +\left[\left.\mathrm{i} L_{N} \frac{f(a ; t)}{W(a ; t)}\right|_{a=\hat{a}} \mid \varrho_{\text {rel }}^{\text {kin-hyd }}\left(x^{N} ; t\right),\right.
\end{aligned}
$$

where $\dot{\hat{n}}_{1}(x)=\mathrm{i} L_{N} \hat{n}_{1}(x), \dot{\hat{H}}^{\mathrm{int}}(\mathbf{r})=\mathrm{i} L_{N} \hat{H}^{\mathrm{int}}(\mathbf{r})$. Having used thereafter the relation

$$
\mathrm{i} L_{N} \hat{f}(a)=\mathrm{i} L_{N} \hat{f}\left(n_{\mathbf{k}}, \mathbf{J}_{\mathbf{k}}, \varepsilon_{\mathbf{k}}\right)=\sum_{\mathbf{k}}\left[\frac{\partial}{\partial \mathbf{n}_{\mathbf{k}}} \hat{\mathbf{f}}(\mathbf{a}) \dot{\hat{\mathbf{n}}}_{\mathbf{k}}+\frac{\partial}{\partial \mathbf{J}_{\mathbf{k}}} \hat{\mathbf{f}}(\mathbf{a}) \dot{\hat{\mathbf{J}}}_{\mathbf{k}}+\frac{\partial}{\partial \varepsilon_{\mathbf{k}}} \hat{\mathbf{f}}(\mathbf{a}) \dot{\hat{\varepsilon}}_{\mathbf{k}}\right],
$$

where $\dot{\hat{n}}_{\mathbf{k}}=\mathrm{i} L_{N} \hat{n}_{\mathbf{k}}, \dot{\hat{\mathbf{J}}}_{\mathbf{k}}=\mathrm{i} L_{N} \hat{\mathbf{J}}_{\mathbf{k}}, \dot{\hat{\varepsilon}}_{\mathbf{k}}=\mathrm{i} L_{N} \hat{\varepsilon}_{\mathbf{k}}$, the last expression in 2.15 can be rewritten in the following form:

$$
\begin{aligned}
{\left[\left.\mathrm{i} L_{N} \frac{f(a ; t)}{W(a ; t)}\right|_{a=\hat{a}}\right] \varrho_{\mathrm{rel}}^{\mathrm{kin}-\mathrm{hyd}}\left(x^{N} ; t\right)=} & \int \mathrm{d} a \sum_{\mathbf{k}}\left\{\dot{\hat{n}}_{\mathbf{k}} W(a ; t)\left[\frac{\partial}{\partial n_{\mathbf{k}}} \frac{f(a ; t)}{W(a ; t)}\right]+\dot{\hat{\mathbf{J}}}_{\mathbf{k}} W(a ; t)\left[\frac{\partial}{\partial \mathbf{J}_{\mathbf{k}}} \frac{f(a ; t)}{W(a ; t)}\right]\right. \\
& \left.+\dot{\hat{\varepsilon}}_{\mathbf{k}} W(a ; t)\left[\frac{\partial}{\partial \varepsilon_{\mathbf{k}}} \frac{f(a ; t)}{W(a ; t)}\right]\right\} \varrho_{\mathrm{L}}\left(x^{N} ; t\right) .
\end{aligned}
$$


Here, we introduced a new relevant distribution function $\varrho_{\mathrm{L}}\left(x^{N}, a ; t\right)$ with the microscopic distribution of large-scale collective variables

$$
\varrho_{\mathrm{L}}\left(x^{N}, a ; t\right)=\varrho_{\mathrm{rel}}^{\mathrm{kin}-\mathrm{hyd}}\left(x^{N} ; t\right) \frac{\hat{f}(a)}{W(a ; t)} .
$$

This relevant distribution function is connected with $\varrho_{\text {rel }}\left(x^{N} ; t\right)$ by the relation

$$
\varrho_{\text {rel }}\left(x^{N} ; t\right)=\int \mathrm{d} a f(a ; t) \varrho_{\mathrm{L}}\left(x^{N}, a ; t\right)
$$

and is obviously normalized to unity

$$
\int \mathrm{d} \Gamma_{N} \varrho_{\mathrm{L}}\left(x^{N} ; t\right)=1
$$

Using the relation (2.17), the average values with a relevant distribution are conveniently represented in the following form:

$$
\langle\ldots\rangle_{\mathrm{rel}}^{t}=\int \mathrm{d} a\langle\ldots\rangle_{\mathrm{L}}^{t} f(a ; t), \quad\langle\ldots\rangle_{\mathrm{L}}^{t}=\int \mathrm{d} \Gamma_{N} \ldots \varrho_{\mathrm{L}}\left(x^{N} ; t\right) .
$$

Now, in accordance with (2.16) and (2.17) we can rewrite the action of the Liouville operator on $\varrho_{\text {rel }}\left(x^{N} ; t\right)$ as follows:

$$
\begin{aligned}
\mathrm{i} L_{N} \varrho_{\text {rel }}\left(x^{N} ; t\right)= & -\int \mathrm{d} a \int \mathrm{d} x \gamma(x ; t) \dot{\hat{n}}_{1}(x) \varrho_{\mathrm{L}}\left(x^{N}, a ; t\right) f(a ; t)-\int \mathrm{d} a \int \mathrm{d} \mathbf{r} \beta(\mathbf{r} ; t) \dot{\hat{H}}^{\mathrm{int}}(\mathbf{r}) \varrho_{\mathrm{L}}\left(x^{N}, a ; t\right) f(a ; t) \\
& +\int \mathrm{d} a \sum_{\mathbf{k}}\left[\dot{\hat{n}}_{\mathbf{k}} W(a ; t) \frac{\partial}{\partial n_{\mathbf{k}}} \frac{f(a ; t)}{W(a ; t)}+W(a ; t) \dot{\hat{\mathbf{J}}}_{\mathbf{k}} \cdot \frac{\partial}{\partial \mathbf{J}_{\mathbf{k}}} \frac{f(a ; t)}{W(a ; t)}\right. \\
& \left.+\dot{\hat{\varepsilon}}_{\mathbf{k}} W(a ; t) \frac{\partial}{\partial \varepsilon_{\mathbf{k}}} \frac{f(a ; t)}{W(a ; t)}\right] \varrho_{\mathrm{L}}\left(x^{N}, a ; t\right)
\end{aligned}
$$

Substituting this expression into [2.6), one obtains, for non-equilibrium distribution function, the following result:

$$
\begin{aligned}
\varrho\left(x^{N} ; t\right)= & \int \mathrm{d} a f(a ; t) \varrho_{\mathrm{L}}\left(x^{N}, a ; t\right) \\
& +\int \mathrm{d} a \int \mathrm{d} \mathbf{r} \int_{-\infty}^{t} \mathrm{~d} t^{\prime} \mathrm{e}^{\epsilon\left(t^{\prime}-t\right)} T_{\mathrm{rel}}\left(t, t^{\prime}\right)\left[1-P_{\mathrm{rel}}\left(t^{\prime}\right)\right] \dot{\hat{H}}^{\mathrm{int}}(\mathbf{r}) \varrho_{\mathrm{L}}\left(x^{N} ; t\right) f\left(a ; t^{\prime}\right) \beta\left(\mathbf{r} ; t^{\prime}\right) \\
& -\int \mathrm{d} a \int \mathrm{d} x \int_{-\infty}^{t} \mathrm{~d} t^{\prime} \mathrm{e}^{\epsilon\left(t^{\prime}-t\right)} T_{\mathrm{rel}}\left(t, t^{\prime}\right)\left[1-P_{\mathrm{rel}}\left(t^{\prime}\right)\right] \dot{\hat{n}}_{1}(x) \varrho_{\mathrm{L}}\left(x^{N}, a ; t^{\prime}\right) f\left(a ; t^{\prime}\right) \gamma\left(x ; t^{\prime}\right) \\
& -\int \mathrm{d} a \sum_{\mathbf{k}} \int_{-\infty}^{t} \mathrm{~d} t^{\prime} \mathrm{e}^{\epsilon\left(t^{\prime}-t\right)} T_{\mathrm{rel}}\left(t, t^{\prime}\right)\left[1-P_{\mathrm{rel}}\left(t^{\prime}\right)\right]\left[\dot{\hat{n}}_{\mathbf{k}} W\left(a ; t^{\prime}\right) \frac{\partial}{\partial n_{\mathbf{k}}} \frac{f\left(a ; t^{\prime}\right)}{W\left(a ; t^{\prime}\right)}\right. \\
& \left.+W\left(a ; t^{\prime}\right) \dot{\hat{\mathbf{J}}}_{\mathbf{k}} \cdot \frac{\partial}{\partial \mathbf{J}_{\mathbf{k}}} \frac{f\left(a ; t^{\prime}\right)}{W\left(a ; t^{\prime}\right)}+\dot{\hat{\varepsilon}}_{\mathbf{k}} W\left(a ; t^{\prime}\right) \frac{\partial}{\partial \varepsilon_{\mathbf{k}}} \frac{f\left(a ; t^{\prime}\right)}{W\left(a ; t^{\prime}\right)}\right] \varrho_{\mathrm{L}}\left(x^{N}, a ; t^{\prime}\right)
\end{aligned}
$$

and the corresponding generalized transport equations:

$$
\begin{aligned}
& \left(\frac{\partial}{\partial t}+\frac{\mathbf{p}}{m} \cdot \frac{\partial}{\partial \mathbf{r}}\right) f_{1}(x ; t)-\int \mathrm{d} x^{\prime} \frac{\partial}{\partial \mathbf{r}} \Phi\left(\left|\mathbf{r}-\mathbf{r}^{\prime}\right|\right) \cdot\left(\frac{\partial}{\partial \mathbf{p}}-\frac{\partial}{\partial \mathbf{p}^{\prime}}\right) g_{2}\left(x, x^{\prime} ; t\right) \\
& =-\int \mathrm{d} \mathbf{r}^{\prime} \int \mathrm{d} a \int_{-\infty}^{t} \mathrm{~d} t^{\prime} \mathrm{e}^{\epsilon\left(t^{\prime}-t\right)} \phi_{n H}\left(x, \mathbf{r}^{\prime}, a ; t, t^{\prime}\right) f\left(a ; t^{\prime}\right) \beta\left(\mathbf{r}^{\prime} ; t^{\prime}\right) \\
& \quad-\int \mathrm{d} x^{\prime} \int \mathrm{d} a \int_{-\infty}^{t} \mathrm{~d} t^{\prime} \mathrm{e}^{\epsilon\left(t^{\prime}-t\right)} \phi_{n n}\left(x, x^{\prime}, a ; t, t^{\prime}\right) f\left(a ; t^{\prime}\right) \gamma\left(x^{\prime} ; t^{\prime}\right) \\
& \quad-\sum_{\mathbf{k}} \int \mathrm{d} a \int_{-\infty}^{t} \mathrm{~d} t^{\prime} \mathrm{e}^{\epsilon\left(t^{\prime}-t\right)}\left[\phi_{n j}\left(x, \mathbf{k}, a ; t, t^{\prime}\right) \cdot \frac{\partial}{\partial \mathbf{J}_{\mathbf{k}}}+\phi_{n \varepsilon}\left(x, \mathbf{k}, a ; t, t^{\prime}\right) \frac{\partial}{\partial \varepsilon_{\mathbf{k}}}\right] \frac{f\left(a ; t^{\prime}\right)}{W\left(a ; t^{\prime}\right)},
\end{aligned}
$$




$$
\begin{aligned}
& \frac{\partial}{\partial t}\left\langle\hat{H}^{\text {int }}(\mathbf{r})\right\rangle^{t}=\left\langle\dot{\hat{H}}^{\text {int }}(\mathbf{r})\right\rangle_{\text {rel }}^{t}-\int \mathrm{d} \mathbf{r}^{\prime} \int \mathrm{d} a \int_{-\infty}^{t} \mathrm{~d} t^{\prime} \mathrm{e}^{\epsilon\left(t^{\prime}-t\right)} \phi_{H H}\left(\mathbf{r}, \mathbf{r}^{\prime}, a ; t, t^{\prime}\right) f\left(a ; t^{\prime}\right) \beta\left(\mathbf{r}^{\prime} ; t^{\prime}\right) \\
& -\int \mathrm{d} x^{\prime} \int \mathrm{d} a \int_{-\infty}^{t} \mathrm{~d} t^{\prime} \mathrm{e}^{\epsilon\left(t^{\prime}-t\right)} \phi_{H n}\left(\mathbf{r}, x^{\prime}, a ; t, t^{\prime}\right) f\left(a ; t^{\prime}\right) \gamma\left(x^{\prime} ; t^{\prime}\right) \\
& -\sum_{\mathbf{k}} \int \mathrm{d} a \int_{-\infty}^{t} \mathrm{~d} t^{\prime} \mathrm{e}^{\epsilon\left(t^{\prime}-t\right)}\left[\phi_{H j}\left(\mathbf{r}, \mathbf{k}, a ; t, t^{\prime}\right) \cdot \frac{\partial}{\partial \mathbf{J}_{\mathbf{k}}}+\phi_{H \varepsilon}\left(\mathbf{r}, \mathbf{k}, a ; t, t^{\prime}\right) \frac{\partial}{\partial \varepsilon_{\mathbf{k}}}\right] \frac{f\left(a ; t^{\prime}\right)}{W\left(a ; t^{\prime}\right)}, \\
& \frac{\partial}{\partial t} f(a ; t)=\sum_{\mathbf{k}}\left[\frac{\partial}{\partial n_{\mathbf{k}}} v_{n}(a ; t)+\frac{\partial}{\partial \mathbf{J}_{\mathbf{k}}} \cdot \mathbf{v}_{j}(a ; t)+\frac{\partial}{\partial \varepsilon_{\mathbf{k}}} v_{\varepsilon}(a ; t)\right] f(a ; t) \\
& =\sum_{\mathbf{k}} \frac{\partial}{\partial \mathbf{J}_{\mathbf{k}}} \cdot \int \mathrm{d} \mathbf{r}^{\prime} \int \mathrm{d} a^{\prime} \int_{-\infty}^{t} \mathrm{~d} t^{\prime} \mathrm{e}^{\epsilon\left(t^{\prime}-t\right)} \phi_{j H}\left(\mathbf{r}^{\prime}, \mathbf{k}, a, a^{\prime} ; t, t^{\prime}\right) f\left(a ; t^{\prime}\right) \beta\left(\mathbf{r}^{\prime} ; t^{\prime}\right) \\
& -\sum_{\mathbf{k}} \frac{\partial}{\partial \varepsilon_{\mathbf{k}}} \int \mathrm{d} \mathbf{r}^{\prime} \int \mathrm{d} a^{\prime} \int_{-\infty}^{t} \mathrm{~d} t^{\prime} \mathrm{e}^{\epsilon\left(t^{\prime}-t\right)} \phi_{\varepsilon H}\left(\mathbf{r}^{\prime}, \mathbf{k}, a, a^{\prime} ; t, t^{\prime}\right) f\left(a ; t^{\prime}\right) \beta\left(\mathbf{r}^{\prime} ; t^{\prime}\right) \\
& +\sum_{\mathbf{k}} \frac{\partial}{\partial \mathbf{J}_{\mathbf{k}}} \cdot \int \mathrm{d} x^{\prime} \int \mathrm{d} a^{\prime} \int_{-\infty}^{t} \mathrm{~d} t^{\prime} \mathrm{e}^{\epsilon\left(t^{\prime}-t\right)} \phi_{j n}\left(x^{\prime}, \mathbf{k}, a, a^{\prime} ; t, t^{\prime}\right) f\left(a ; t^{\prime}\right) \gamma\left(x^{\prime} ; t^{\prime}\right) \\
& -\sum_{\mathbf{k}} \frac{\partial}{\partial \varepsilon_{\mathbf{k}}} \int \mathrm{d} x^{\prime} \int \mathrm{d} a^{\prime} \int_{-\infty}^{t} \mathrm{~d} t^{\prime} \mathrm{e}^{\epsilon\left(t^{\prime}-t\right)} \phi_{\varepsilon n}\left(x^{\prime}, \mathbf{k}, a, a^{\prime} ; t, t^{\prime}\right) f\left(a ; t^{\prime}\right) \gamma\left(x^{\prime} ; t^{\prime}\right) \\
& +\sum_{\mathbf{k}, \mathbf{q}} \int \mathrm{d} a^{\prime} \int_{-\infty}^{t} \mathrm{~d} t^{\prime} \mathrm{e}^{\epsilon\left(t^{\prime}-t\right)} \frac{\partial}{\partial \mathbf{J}_{\mathbf{k}}} \cdot \phi_{j j}\left(\mathbf{k}, \mathbf{q}, a, a^{\prime} ; t, t^{\prime}\right) \cdot \frac{\partial}{\partial \mathbf{J}_{\mathbf{q}}} \frac{f\left(a ; t^{\prime}\right)}{W\left(a ; t^{\prime}\right)} \\
& +\sum_{\mathbf{k}, \mathbf{q}} \int \mathrm{d} a^{\prime} \int_{-\infty}^{t} \mathrm{~d} t^{\prime} \mathrm{e}^{\epsilon\left(t^{\prime}-t\right)} \frac{\partial}{\partial \varepsilon_{\mathbf{k}}} \phi_{\varepsilon \varepsilon}\left(\mathbf{k}, \mathbf{q}, a, a^{\prime} ; t, t^{\prime}\right) \frac{\partial}{\partial \varepsilon_{\mathbf{q}}} \frac{f\left(a ; t^{\prime}\right)}{W\left(a ; t^{\prime}\right)} \\
& +\sum_{\mathbf{k}, \mathbf{q}} \int \mathrm{d} a^{\prime} \int_{-\infty}^{t} \mathrm{~d} t^{\prime} \mathrm{e}^{\epsilon\left(t^{\prime}-t\right)}\left[\frac{\partial}{\partial \mathbf{J}_{\mathbf{k}}} \cdot \phi_{j \varepsilon}\left(\mathbf{k}, \mathbf{q}, a, a^{\prime} ; t, t^{\prime}\right) \frac{\partial}{\partial \varepsilon_{\mathbf{q}}}+\frac{\partial}{\partial \varepsilon_{\mathbf{k}}} \phi_{\varepsilon j}\left(\mathbf{k}, \mathbf{q}, a, a^{\prime} ; t, t^{\prime}\right) \cdot \frac{\partial}{\partial \mathbf{J}_{\mathbf{q}}}\right] \frac{f\left(a ; t^{\prime}\right)}{W\left(a ; t^{\prime}\right)} .
\end{aligned}
$$

The generalized transport equations (2.23), (2.24) include a relevant two-particle distribution function of particles $g_{2}\left(x, x^{\prime} ; t\right)$ :

$$
g_{2}\left(x, x^{\prime} ; t\right)=\int \mathrm{d} \Gamma_{N-2} \varrho_{\text {rel }}\left(x^{N} ; t\right)=\left\langle\hat{n}_{1}(x) \hat{n}_{1}\left(x^{\prime}\right)\right\rangle_{\text {rel }}^{t}=\left\langle\hat{G}\left(x, x^{\prime}\right)\right\rangle_{\text {rel }}^{t}=\int \mathrm{d} a g_{2}^{\mathrm{L}}\left(x, x^{\prime} ; a ; t\right) f(a ; t),
$$

where $\hat{G}\left(x, x^{\prime}\right)=\hat{n}_{1}(x) \hat{n}_{1}\left(x^{\prime}\right)$, and $g_{2}^{\mathrm{L}}\left(x, x^{\prime} ; a ; t\right)=\int \mathrm{d} \Gamma_{N-2} \varrho_{\mathrm{L}}\left(x^{N} ; a ; t\right)$ is the two-particle relevant distribution function of large-scale collective variables. The generalized transport kernels

$$
\phi_{\alpha \beta}\left(t, t^{\prime}\right)=\left\langle I_{\alpha}(t) T_{\text {rel }}\left(t, t^{\prime}\right) I_{\beta}\left(t^{\prime}\right)\right\rangle_{\mathrm{L}}^{t^{\prime}}, \quad \alpha, \beta=\{n, H, \mathbf{j}, \varepsilon\},
$$

that describe non-Markovian kinetic and hydrodynamic processes, are non-equilibrium correlation functions of generalized fluxes $I_{\alpha}, I_{\beta}$ :

$$
\begin{aligned}
& \hat{I}_{n}(x ; t)=[1-P(t)] \dot{\hat{n}}_{1}(x), \quad \hat{I}_{H}(\mathbf{r} ; t)=[1-P(t)] \dot{\hat{H}}^{\text {int }}(\mathbf{r}),
\end{aligned}
$$

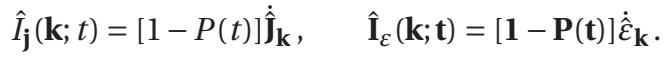

Here, $P(t)$ is the generalized Mori operator related to Kawasaki-Gunton projection operator $P_{\text {rel }}(t)$ by the following relation

$$
P_{\text {rel }}(t) a(x) \varrho_{\text {rel }}\left(x^{N} ; t\right)=\varrho_{\text {rel }}\left(x^{N} ; t\right) P(t) a(x) .
$$


It should be emphasized that in 2.26 the averages are calculated with a distribution function $\varrho_{\mathrm{L}}\left(x^{N}, a ; t\right)$ (2.20), so that the transport kernels are functions of collective variables $a_{\mathbf{k}}$. In equation [2.25], the functions (called hydrodynamic velocities) $v_{n, \mathbf{k}}(a ; t), \mathbf{v}_{j, \mathbf{k}}(a ; t), v_{\varepsilon, \mathbf{k}}(a ; t)$ represent the fluxes in the space of collective variables and are defined as:

$$
\begin{array}{r}
v_{n}(a ; t)=\int \mathrm{d} \Gamma_{N} \dot{\hat{n}}_{\mathbf{k}} \varrho_{\mathrm{L}}\left(x^{N}, a ; t\right)=\left\langle\dot{\hat{n}}_{\mathbf{k}}\right\rangle_{\mathrm{L}}^{t}, \\
v_{j}(a ; t)=\int \mathrm{d} \Gamma_{N} \dot{\hat{\mathbf{J}}}_{\mathbf{k}} \varrho_{\mathrm{L}}\left(x^{N}, a ; t\right)=\left\langle\dot{\hat{J}_{\mathbf{k}}}\right\rangle_{\mathrm{L}}^{t}, \\
v_{\varepsilon}(a ; t)=\int \mathrm{d} \Gamma_{N} \dot{\hat{\varepsilon}}_{\mathbf{k}} \varrho_{\mathrm{L}}\left(x^{N}, a ; t\right)=\left\langle\dot{\hat{\varepsilon}_{\mathbf{k}}}\right\rangle_{\mathrm{L}}^{t} .
\end{array}
$$

The presented system of transport equations provides a consistent description of kinetic and hydrodynamic processes of classical fluids which take into account long-living fluctuations.

The system of transport equations 2.23-2.25 is not closed due to Lagrange parameters $\gamma(x ; t)$, $\beta\left(\mathbf{r}^{\prime} ; t^{\prime}\right)$, which are determined from the corresponding self-consistent conditions.

To describe the kinetics of heterophase fluctuations, as discussed in the Introduction, in addition to the equation for non-equilibrium one-particle distribution function it is important to consider the equations for higher non-equilibrium distribution functions of groups of interacting particles. The kinetics of these phases is described by kinetic equations for higher non-equilibrium distribution functions of finite number of particles in heterophase formations. Therefore, to describe these heterophase kinetic processes we must supplement this system of transport equations with the kinetic equation $f_{2}\left(x, x^{\prime} ; t\right)$, and hence for $f_{s}\left(x_{1} \ldots x_{s} ; t\right), s<N$ :

$$
\begin{aligned}
& \frac{\partial}{\partial t} f_{2}\left(x, x^{\prime} ; t\right)+\mathrm{i} L_{2} f_{2}\left(x, x^{\prime} ; t\right)-\int \mathrm{d} x^{\prime \prime}[\mathrm{i} L(13)+\mathrm{i} L(23)] f_{3}\left(x, x^{\prime}, x^{\prime \prime} ; t\right) \\
& =\mathrm{i} L_{2} \Delta f_{2}\left(x, x^{\prime} ; t\right)-\int d x^{\prime \prime}[\mathrm{i} L(13)+\mathrm{i} L(23)] \Delta f_{3}\left(x, x^{\prime}, x^{\prime \prime} ; t\right) \\
& \quad+\int \mathrm{d} x^{\prime \prime} \int \mathrm{d} a \int_{-\infty}^{t} \mathrm{~d} t^{\prime} \mathrm{e}^{\epsilon\left(t^{\prime}-t\right)} \phi_{G n}\left(x, x^{\prime}, x^{\prime \prime}, a ; t, t^{\prime}\right) f\left(a ; t^{\prime}\right) \gamma\left(x^{\prime \prime} ; t^{\prime}\right) \\
& \quad-\int \mathrm{d} \mathbf{r}^{\prime \prime} \int \mathrm{d} a \int_{-\infty}^{t} \mathrm{~d} t^{\prime} \mathrm{e}^{\epsilon\left(t^{\prime}-t\right)} \phi_{G H}\left(x, x^{\prime}, \mathbf{r}^{\prime \prime}, a ; t, t^{\prime}\right) f\left(a ; t^{\prime}\right) \beta\left(\mathbf{r}^{\prime \prime} ; t^{\prime}\right) \\
& \quad-\sum_{\mathbf{k}} \int \mathrm{d} a \int_{-\infty}^{t} \mathrm{~d} t^{\prime} \mathrm{e}^{\epsilon\left(t^{\prime}-t\right)}\left[\phi_{G j}\left(x, x^{\prime}, \mathbf{k}, a ; t, t^{\prime}\right) \cdot \frac{\partial}{\partial \mathbf{J}_{\mathbf{k}}}+\phi_{G \varepsilon}\left(x, x^{\prime}, \mathbf{k}, a ; t, t^{\prime}\right) \frac{\partial}{\partial \varepsilon_{\mathbf{k}}}\right] \frac{f\left(a ; t^{\prime}\right)}{W\left(a ; t^{\prime}\right)}, \\
& \frac{\partial}{\partial t} f_{s}\left(x^{s} ; t\right)+\mathrm{i} L_{s} f_{s}\left(x^{s} ; t\right)-\sum_{j} \frac{1}{s !} \int \mathrm{d} x_{s+1} \mathrm{i} L(j, s+1) f_{s+1}\left(x^{s}, x_{s+1} ; t\right) \\
& =\mathrm{i} L_{s} \Delta f_{s}\left(x^{s} ; t\right)-\sum_{j} \frac{1}{s !} \int d x_{s+1} \mathrm{i} L(j, s+1) \Delta f_{s+1}\left(x^{s}, x_{s+1} ; t\right) \\
& \quad+\int \mathrm{d} x^{\prime \prime} \int \mathrm{d} a \int_{-\infty}^{t} \mathrm{~d} t^{\prime} \mathrm{e}^{\epsilon\left(t^{\prime}-t\right)} \phi_{G_{s} n}\left(x^{s}, x^{\prime \prime}, a ; t, t^{\prime}\right) f\left(a ; t^{\prime}\right) \gamma\left(x^{\prime \prime} ; t^{\prime}\right) \\
& \quad-\int \mathrm{d} \mathbf{r}^{\prime \prime} \int_{-\infty}^{t} \mathrm{~d} a \int_{-\infty}^{t} \mathrm{~d} t^{\prime} \mathrm{e}^{\epsilon\left(t^{\prime}-t\right)} \phi_{G_{s} H}\left(x^{s}, \mathbf{r}^{\prime \prime}, a ; t, t^{\prime}\right) f\left(a ; t^{\prime}\right) \beta\left(\mathbf{r}^{\prime \prime} ; t^{\prime}\right) \\
& \quad-\sum_{\mathbf{k}} \int \mathrm{d} a \int_{-\infty}^{t} \mathrm{~d} t^{\prime} \mathrm{e}^{\epsilon\left(t^{\prime}-t\right)}\left[\phi_{G_{s} j}\left(x^{s}, \mathbf{k}, a ; t, t^{\prime}\right) \cdot \frac{\partial}{\partial \mathbf{J}_{\mathbf{k}}}+\phi_{G_{s} \varepsilon}\left(x^{s}, \mathbf{k}, a ; t, t^{\prime}\right) \frac{\partial}{\partial \varepsilon_{\mathbf{k}}}\right] \frac{f\left(a ; t^{\prime}\right)}{W\left(a ; t^{\prime}\right)},
\end{aligned}
$$

where

$$
\Delta f_{2}\left(x, x^{\prime} ; t\right)=f_{2}\left(x, x^{\prime} ; t\right)-g_{2}\left(x, x^{\prime} ; t\right), \quad \Delta f_{s}\left(x^{s} ; t\right)=f_{s}\left(x^{s} ; t\right)-g_{s}\left(x^{s} ; t\right) .
$$


In equation 2.31), the two-particle Liouville operator

$$
\mathrm{i} L_{2}=\mathrm{i} L_{0}(x)+i L_{0}\left(x^{\prime}\right)+\mathrm{i} L\left(x, x^{\prime}\right)
$$

was introduced. It contains a one-particle operator

$$
\mathrm{i} L_{0}(x)=\frac{\mathbf{p}}{m} \cdot \frac{\partial}{\partial \mathbf{r}}, \quad x=\{\mathbf{r}, \mathbf{p}\}
$$

as well as a potential part

$$
\mathrm{i} L\left(x, x^{\prime}\right)=\frac{\partial}{\partial \mathbf{r}} \Phi\left(\left|\mathbf{r}-\mathbf{r}^{\prime}\right|\right) \cdot\left(\frac{\partial}{\partial \mathbf{p}}-\frac{\partial}{\partial \mathbf{p}^{\prime}}\right) .
$$

Accordingly, in equation (2.32), $\mathrm{i} L_{s}$ is the $s$-particle Liouville operator,

$$
g_{s}\left(x_{1} \ldots x_{s} ; t\right)=\left\langle\hat{G}_{s}\left(x_{1} \ldots x_{s}\right)\right\rangle_{\mathrm{rel}}^{t}=\int \mathrm{d} a g_{s}^{\mathrm{L}}\left(x_{1} \ldots x_{s} ; a ; t\right) f(a ; t),
$$

where

$$
g_{s}^{\mathrm{L}}\left(x_{1} \ldots x_{s} ; a ; t\right)=\int \mathrm{d} \Gamma_{N} \hat{G}_{s}\left(x_{1} \ldots x_{s}\right) \varrho_{\mathrm{L}}\left(x^{N} ; a ; t\right)
$$

is the $s$-particle relevant distribution function of large-scale variables and $\hat{G}_{s}\left(x^{s}\right)=\hat{n}_{1}\left(x_{1}\right) \ldots \hat{n}_{1}\left(x_{s}\right)$.

Thus, we obtained a system of equations for non-equilibrium one-, two-, s-particle distribution functions which take into account nonlinear hydrodynamic fluctuations.

Now, we discuss the equation 2.25 that is of Fokker-Planck type for a non-equilibrium distribution function of collective variables which take into account the kinetic processes. The transport kernel in this equation $\phi_{n n}\left(x, x^{\prime} ; t, t^{\prime}\right)$ describes a dissipation of kinetic processes, while the kernels $\phi_{n j}\left(x, \mathbf{k}, a ; t, t^{\prime}\right)$, $\phi_{n \varepsilon}\left(x, \mathbf{k}, a ; t, t^{\prime}\right), \phi_{j n}\left(x, \mathbf{k}, a ; t, t^{\prime}\right), \phi_{\varepsilon n}\left(x, \mathbf{k}, a ; t, t^{\prime}\right)$ describe a dissipation of correlations between kinetic and hydrodynamic processes. To uncover a more detailed structure of transport kernels $\phi_{n n}\left(x ; x^{\prime}, a ; t, t^{\prime}\right)$, $\phi_{G n}\left(x ; x^{\prime}, x^{\prime \prime}, a ; t, t^{\prime}\right)$ we consider the action of Liouville operator on $\hat{n}_{1}(x)$ and $\hat{G}\left(x, x^{\prime}\right)$ :

$$
\begin{gathered}
\mathrm{i} L_{N} \hat{n}_{1}(x)=-\frac{\partial}{\partial \mathbf{r}} \cdot \frac{1}{m} \hat{\mathbf{j}}(\mathbf{r}, \mathbf{p})+\frac{\partial}{\partial \mathbf{p}} \cdot \hat{\mathbf{F}}(\mathbf{r}, \mathbf{p}), \\
\mathrm{i} L_{N} \hat{G}\left(x, x^{\prime}\right)=-\frac{\partial}{\partial \mathbf{r}} \cdot \frac{1}{m} \hat{\mathbf{j}}(\mathbf{r}, \mathbf{p}) \hat{n}_{1}\left(x^{\prime}\right)-\hat{n}_{1}(x) \frac{\partial}{\partial \mathbf{r}^{\prime}} \cdot \frac{1}{m} \hat{\mathbf{j}}\left(\mathbf{r}^{\prime}, \mathbf{p}^{\prime}\right)+\frac{\partial}{\partial \mathbf{p}} \cdot \hat{\mathbf{F}}(\mathbf{r}, \mathbf{p}) \hat{n}_{1}\left(x^{\prime}\right)+\hat{n}_{1}(x) \frac{\partial}{\partial \mathbf{p}^{\prime}} \cdot \hat{\mathbf{F}}\left(\mathbf{r}^{\prime}, \mathbf{p}^{\prime}\right),
\end{gathered}
$$

where

$$
\hat{\mathbf{j}}(\mathbf{r}, \mathbf{p})=\sum_{j=1}^{N} \mathbf{p}_{j} \delta\left(\mathbf{r}-\mathbf{r}_{j}\right) \delta\left(\mathbf{p}-\mathbf{p}_{j}\right)
$$

is the microscopic density of the momentum vector in coordinate-momentum space,

$$
\hat{\mathbf{F}}(\mathbf{r}, \mathbf{p})=\sum_{l \neq j} \frac{\partial}{\partial \mathbf{r}_{j}} \Phi\left(\left|\mathbf{r}_{j}-\mathbf{r}_{l}\right|\right) \delta\left(\mathbf{r}-\mathbf{r}_{j}\right) \delta\left(\mathbf{p}-\mathbf{p}_{j}\right)
$$

is the microscopic density of force vector in coordinate-momentum space. Taking into account the equations 2.33-2.36 for the kinetic transport kernels, we obtain:

$$
\begin{aligned}
\phi_{n n}\left(x ; x^{\prime}, a ; t, t^{\prime}\right)= & -\left[\frac{\partial}{\partial \mathbf{r}} \cdot D_{j j}\left(x, x^{\prime}, a ; t, t^{\prime}\right) \cdot \frac{\partial}{\partial \mathbf{r}^{\prime}}-\frac{\partial}{\partial \mathbf{p}} \cdot D_{F j}\left(x, x^{\prime}, a ; t, t^{\prime}\right) \cdot \frac{\partial}{\partial \mathbf{r}^{\prime}}\right. \\
& \left.-\frac{\partial}{\partial \mathbf{r}} \cdot D_{j F}\left(x, x^{\prime}, a ; t, t^{\prime}\right) \cdot \frac{\partial}{\partial \mathbf{p}^{\prime}}+\frac{\partial}{\partial \mathbf{p}} \cdot D_{F F}\left(x, x^{\prime}, a ; t, t^{\prime}\right) \cdot \frac{\partial}{\partial \mathbf{p}^{\prime}}\right],
\end{aligned}
$$

where

$$
\begin{aligned}
D_{j j}\left(x, x^{\prime}, a ; t, t^{\prime}\right) & =\int \mathrm{d} \Gamma_{N} \hat{\mathbf{j}}(x) T\left(t, t^{\prime}\right)\left[1-P\left(t^{\prime}\right)\right] \hat{\mathbf{j}}\left(x^{\prime}\right) \rho_{\mathrm{L}}\left(x^{N} ; t^{\prime}\right), \\
D_{F F}\left(x, x^{\prime}, a ; t, t^{\prime}\right) & =\int \mathrm{d} \Gamma_{N} \hat{\mathbf{F}}(x) T\left(t, t^{\prime}\right)\left[1-P\left(t^{\prime}\right)\right] \hat{\mathbf{F}}\left(x^{\prime}\right) \rho_{\mathrm{L}}\left(x^{N} ; t^{\prime}\right)
\end{aligned}
$$


are the generalized diffusion and the particle friction coefficients in the coordinate-momentum space. Moreover,

$$
\int \mathrm{d} \mathbf{p} \int \mathrm{d} \mathbf{p}^{\prime} D_{j j}\left(x, x^{\prime} ; t, t^{\prime}\right)=D_{j j}\left(\mathbf{r}, \mathbf{r}^{\prime} ; t, t^{\prime}\right), \quad \int \mathrm{d} \mathbf{p} \int \mathrm{d} \mathbf{p}^{\prime} D_{F F}\left(x, x^{\prime} ; t, t^{\prime}\right)=D_{F F}\left(\mathbf{r}, \mathbf{r}^{\prime} ; t, t^{\prime}\right)
$$

are the generalized coefficients of diffusion and friction. Similarly, we obtain the expression for the transport kernel $\phi_{G n}\left(x ; x^{\prime}, x^{\prime \prime} ; t, t^{\prime}\right)$ :

$$
\begin{aligned}
\phi_{G n}\left(x ; x^{\prime}, x^{\prime \prime}, a ; t, t^{\prime}\right)= & -\left[\frac{\partial}{\partial \mathbf{r}} \cdot D_{j j n}\left(x, x^{\prime}, x^{\prime \prime}, a ; t, t^{\prime}\right) \cdot \frac{\partial}{\partial \mathbf{r}^{\prime}}+\frac{\partial}{\partial \mathbf{r}} \cdot D_{j n j}\left(x, x^{\prime}, x^{\prime \prime}, a ; t, t^{\prime}\right) \cdot \frac{\partial}{\partial \mathbf{r}^{\prime \prime}}\right. \\
& -\frac{\partial}{\partial \mathbf{p}} \cdot D_{F j n}\left(x, x^{\prime}, x^{\prime \prime}, a ; t, t^{\prime}\right) \cdot \frac{\partial}{\partial \mathbf{r}^{\prime}}-\frac{\partial}{\partial \mathbf{p}} \cdot D_{F n j}\left(x, x^{\prime}, x^{\prime \prime}, a ; t, t^{\prime}\right) \cdot \frac{\partial}{\partial \mathbf{r}^{\prime \prime}} \\
& -\frac{\partial}{\partial \mathbf{r}} \cdot D_{j F n}\left(x, x^{\prime}, x^{\prime \prime}, a ; t, t^{\prime}\right) \cdot \frac{\partial}{\partial \mathbf{p}^{\prime}}-\frac{\partial}{\partial \mathbf{r}} \cdot D_{j n F}\left(x, x^{\prime}, x^{\prime \prime}, a ; t, t^{\prime}\right) \cdot \frac{\partial}{\partial \mathbf{p}^{\prime \prime}} \\
& \left.+\frac{\partial}{\partial \mathbf{p}} \cdot D_{F F n}\left(x, x^{\prime}, x^{\prime \prime}, a ; t, t^{\prime}\right) \cdot \frac{\partial}{\partial \mathbf{p}^{\prime}}+\frac{\partial}{\partial \mathbf{p}} \cdot D_{F n F}\left(x, x^{\prime}, x^{\prime \prime}, a ; t, t^{\prime}\right) \cdot \frac{\partial}{\partial \mathbf{p}^{\prime \prime}}\right] .
\end{aligned}
$$

It is remarkable that the expression

$$
\int \mathrm{d} x^{\prime} \int \mathrm{d} a \int_{-\infty}^{t} \mathrm{~d} t^{\prime} \mathrm{e}^{\epsilon\left(t^{\prime}-t\right)} \phi_{n n}\left(x, x^{\prime}, a ; t, t^{\prime}\right) f\left(a ; t^{\prime}\right) \gamma\left(x^{\prime} ; t^{\prime}\right)
$$

in equation 2.23) with 2.37) is the generalized collision integral of Fokker-Planck type in the coordinatemomentum space. That is, taking into account (2.26) and (2.37), the kinetic equation (2.23) can be written as follows:

$$
\begin{aligned}
& \left(\frac{\partial}{\partial t}+\frac{\mathbf{p}}{m} \frac{\partial}{\partial \mathbf{r}}\right) f_{1}(x ; t)-\int \mathrm{d} x^{\prime} \int \mathrm{d} a \frac{\partial}{\partial \mathbf{r}} \Phi\left(\left|\mathbf{r}-\mathbf{r}^{\prime}\right|\right)\left(\frac{\partial}{\partial \mathbf{p}}-\frac{\partial}{\partial \mathbf{p}^{\prime}}\right) g_{2}^{l}\left(x, x^{\prime}, a ; t\right) f(a ; t) \\
& =-\int \mathrm{d} \mathbf{r}^{\prime} \int \mathrm{d} a \int_{-\infty}^{t} \mathrm{~d} t^{\prime} \mathrm{e}^{\epsilon\left(t^{\prime}-t\right)} \phi_{n H}\left(x, \mathbf{r}^{\prime}, a ; t, t^{\prime}\right) f\left(a ; t^{\prime}\right) \beta\left(\mathbf{r}^{\prime} ; t^{\prime}\right) \\
& \quad-\int \mathrm{d} x^{\prime} \int \mathrm{d} a \int_{-\infty}^{t} \mathrm{~d} t^{\prime} \mathrm{e}^{\epsilon\left(t^{\prime}-t\right)} \frac{\partial}{\partial \mathbf{r}} \cdot D_{j j}\left(x, x^{\prime}, a ; t, t^{\prime}\right) \cdot \frac{\partial}{\partial \mathbf{r}^{\prime}} \gamma\left(x^{\prime} ; t^{\prime}\right) f\left(a ; t^{\prime}\right) \\
& \quad+\int \mathrm{d} x^{\prime} \int \mathrm{d} a \int_{-\infty}^{t} \mathrm{~d} t^{\prime} \mathrm{e}^{\epsilon\left(t^{\prime}-t\right)}\left[\frac{\partial}{\partial \mathbf{p}} \cdot D_{F j}\left(x, x^{\prime}, a ; t, t^{\prime}\right) \cdot \frac{\partial}{\partial \mathbf{r}^{\prime}}\right. \\
& \left.\quad+\frac{\partial}{\partial \mathbf{r}} \cdot D_{j F}\left(x, x^{\prime}, a ; t, t^{\prime}\right) \cdot \frac{\partial}{\partial \mathbf{p}^{\prime}}-\frac{\partial}{\partial \mathbf{p}} \cdot D_{F F}\left(x, x^{\prime}, a ; t, t^{\prime}\right) \cdot \frac{\partial}{\partial \mathbf{p}^{\prime}}\right] \gamma\left(x^{\prime} ; t^{\prime}\right) f\left(a ; t^{\prime}\right) \\
& \quad-\sum_{\mathbf{k}} \int \mathrm{d} a \int_{-\infty}^{t} \mathrm{~d} t^{\prime} \mathrm{e}^{\epsilon\left(t^{\prime}-t\right)}\left[\phi_{n j}\left(x, \mathbf{k}, a ; t, t^{\prime}\right) \cdot \frac{\partial}{\partial \mathbf{J}_{\mathbf{k}}}+\phi_{n \varepsilon}\left(x, \mathbf{k}, a ; t, t^{\prime}\right) \frac{\partial}{\partial \varepsilon_{\mathbf{k}}}\right] \frac{f\left(a ; t^{\prime}\right)}{W\left(a ; t^{\prime}\right)}
\end{aligned}
$$

In the equation (2.25), the quantities $\phi_{j j}\left(\mathbf{k}, \mathbf{q}, a, a^{\prime} ; t, t^{\prime}\right), \quad \phi_{j \varepsilon}\left(\mathbf{k}, \mathbf{q}, a, a^{\prime} ; t, t^{\prime}\right), \quad \phi_{\varepsilon j}\left(\mathbf{k}, \mathbf{q}, a, a^{\prime} ; t, t^{\prime}\right)$, $\phi_{\varepsilon \varepsilon}\left(\mathbf{k}, \mathbf{q}, a, a^{\prime} ; t, t^{\prime}\right)$ correspond to the dissipative processes connected with the correlations between viscous and heat hydrodynamic processes. Note that the equations [2.31, 2.32) have the structure similar to the equation (2.39) but with more complex many-particle transport kernels. Thus, we obtained a system of equations for non-equilibrium one-, two-, s-particle distribution functions on collision integral of Fokker-Planck type which take into account nonlinear hydrodynamic fluctuations.

The set of equations (2.23), 2.25), (2.31), 2.32) allow for two limiting cases. The first, if the description of non-equilibrium processes does not take into account nonlinear hydrodynamic fluctuations, we will obtain the system of equations for a consistent description of kinetics and hydrodynamics obtained in 
papers [39, 42, 43]. These systems can be written as follows:

$$
\begin{gathered}
\left(\frac{\partial}{\partial t}+\frac{\mathbf{p}}{m} \cdot \frac{\partial}{\partial \mathbf{r}}\right) f_{1}(x ; t)-\int \mathrm{d} x^{\prime} \frac{\partial}{\partial \mathbf{r}} \Phi\left(\left|\mathbf{r}-\mathbf{r}^{\prime}\right|\right) \cdot\left(\frac{\partial}{\partial \mathbf{p}}-\frac{\partial}{\partial \mathbf{p}^{\prime}}\right) g_{2}\left(x, x^{\prime} ; t\right) \\
=-\int \mathrm{d} \mathbf{r}^{\prime} \int_{-\infty}^{t} \mathrm{~d} t^{\prime} \mathrm{e}^{\epsilon\left(t^{\prime}-t\right)} \phi_{n H}\left(x, \mathbf{r}^{\prime} ; t, t^{\prime}\right) \beta\left(\mathbf{r}^{\prime} ; t^{\prime}\right)-\int \mathrm{d} x^{\prime} \int_{-\infty}^{t} \mathrm{~d} t^{\prime} \mathrm{e}^{\epsilon\left(t^{\prime}-t\right)} \phi_{n n}\left(x, x^{\prime} ; t, t^{\prime}\right) \gamma\left(x^{\prime} ; t^{\prime}\right) \\
\frac{\partial}{\partial t}\left\langle\hat{H}^{\text {int }}(\mathbf{r})\right\rangle^{t}=\left\langle\dot{\hat{H}}^{\text {int }}(\mathbf{r})\right\rangle_{\mathrm{rel}}^{t}-\int \mathrm{d} \mathbf{r}^{\prime} \int_{-\infty}^{t} \mathrm{~d} t^{\prime} \mathrm{e}^{\epsilon\left(t^{\prime}-t\right)} \phi_{H H}\left(\mathbf{r}, \mathbf{r}^{\prime} ; t, t^{\prime}\right) \beta\left(\mathbf{r}^{\prime} ; t^{\prime}\right) \\
-\int \mathrm{d} x^{\prime} \int_{-\infty}^{t} \mathrm{~d} t^{\prime} \mathrm{e}^{\epsilon\left(t^{\prime}-t\right)} \phi_{H n}\left(\mathbf{r}, x^{\prime} ; t, t^{\prime}\right) \gamma\left(x^{\prime} ; t^{\prime}\right)
\end{gathered}
$$

where the transport kernels $\phi_{n n}\left(x, x^{\prime} ; t, t^{\prime}\right), \phi_{n H}\left(x, \mathbf{r}^{\prime} ; t, t^{\prime}\right), \phi_{H n}\left(\mathbf{r}, x^{\prime} ; t, t^{\prime}\right), \phi_{H H}\left(\mathbf{r}, \mathbf{r}^{\prime} ; t, t^{\prime}\right)$ are given by expression 2.27). An averaging in these kernels is performed using the relevant statistical operator $\varrho_{\text {rel }}^{\text {kin-hyd }}\left(x^{N} ; t\right)$. If in equations [2.40), 2.41) we do not consider the contribution from the potential energy of interaction, which is true for a dilute gas, then we shall obtain a generalized kinetic equation for a non-equilibrium one-particle distribution function [44]:

$$
\begin{aligned}
& \left(\frac{\partial}{\partial t}+\frac{\mathbf{p}}{m} \cdot \frac{\partial}{\partial \mathbf{r}}\right) f_{1}(x ; t)-\int \mathrm{d} x^{\prime} \frac{\partial}{\partial \mathbf{r}} \Phi\left(\left|\mathbf{r}-\mathbf{r}^{\prime}\right|\right)\left(\frac{\partial}{\partial \mathbf{p}}-\frac{\partial}{\partial \mathbf{p}^{\prime}}\right) g_{2}\left(x, x^{\prime} ; t\right) \\
& =\int \mathrm{d} x^{\prime} \int_{-\infty}^{t} \mathrm{~d} t^{\prime} \mathrm{e}^{\epsilon\left(t^{\prime}-t\right)} \phi_{n n}\left(x, x^{\prime} ; t, t^{\prime}\right) \gamma\left(x^{\prime} ; t^{\prime}\right) .
\end{aligned}
$$

The second, if we do not take into account the kinetic processes, then we shall obtain generalized (non-Markov) Fokker-Planck equation for non-equilibrium distribution function of collective variables, which can be obtained using the method of Zwanzig projection operators or using the method of Zubarev non-equilibrium statistical operator [45]:

$$
\begin{aligned}
\frac{\partial}{\partial t} f(a ; t)= & \sum_{\mathbf{k}}\left[\frac{\partial}{\partial n_{\mathbf{k}}} v_{n}(a ; t)+\frac{\partial}{\partial \mathbf{J}_{\mathbf{k}}} \cdot \mathbf{v}_{j}(a ; t)+\frac{\partial}{\partial \varepsilon_{\mathbf{k}}} v_{\varepsilon}(a ; t)\right] f(a ; t) \\
= & \sum_{\mathbf{k}, \mathbf{q}} \int \mathrm{d} a^{\prime} \int_{-\infty}^{t} \mathrm{~d} t^{\prime} \mathrm{e}^{\epsilon\left(t^{\prime}-t\right)} \frac{\partial}{\partial \mathbf{J}_{\mathbf{k}}} \cdot \phi_{j j}\left(\mathbf{k}, \mathbf{q}, a, a^{\prime} ; t, t^{\prime}\right) \cdot \frac{\partial}{\partial \mathbf{J}_{\mathbf{q}}} \frac{f\left(a ; t^{\prime}\right)}{W\left(a ; t^{\prime}\right)} \\
& +\sum_{\mathbf{k}, \mathbf{q}} \int \mathrm{d} a^{\prime} \int_{-\infty}^{t} \mathrm{~d} t^{\prime} \mathrm{e}^{\epsilon\left(t^{\prime}-t\right)} \frac{\partial}{\partial \varepsilon_{\mathbf{k}}} \phi_{\varepsilon \varepsilon}\left(\mathbf{k}, \mathbf{q}, a, a^{\prime} ; t, t^{\prime}\right) \frac{\partial}{\partial \varepsilon_{\mathbf{q}}} \frac{f\left(a ; t^{\prime}\right)}{W\left(a ; t^{\prime}\right)}+\sum_{\mathbf{k}, \mathbf{q}} \int \mathrm{d} a^{\prime} \int_{-\infty}^{t} \mathrm{~d} t^{\prime} \mathrm{e}^{\epsilon\left(t^{\prime}-t\right)} \\
& \times\left[\frac{\partial}{\partial \mathbf{J}_{\mathbf{k}}} \cdot \phi_{j \varepsilon}\left(\mathbf{k}, \mathbf{q}, a, a^{\prime} ; t, t^{\prime}\right) \frac{\partial}{\partial \varepsilon_{\mathbf{q}}}+\frac{\partial}{\partial \varepsilon_{\mathbf{k}}} \phi_{\varepsilon j}\left(\mathbf{k}, \mathbf{q}, a, a^{\prime} ; t, t^{\prime}\right) \cdot \frac{\partial}{\partial \mathbf{J}_{\mathbf{q}}}\right] \frac{f\left(a ; t^{\prime}\right)}{W\left(a ; t^{\prime}\right)} .
\end{aligned}
$$

One of the main problems for the analysis of transport equations (2.23)-2.25) and transport kernels are the calculations of the structure functions $W(a ; t)$ of collective variables and of hydrodynamic velocities $v_{n}(a ; t), v_{j}(a ; t), v_{\varepsilon}(a ; t)$. We consider one of these ways in the next section.

\section{Calculation of structure function $W(a ; t)$ and hydrodynamical veloci- ties $v_{\alpha}(a ; t)$ by the collective variable method}

In the Kawasaki theory [46] of non-linear fluctuations, the structure function is approximated by a Gaussian dependence on collective variables. In this case, as it can be seen, the hydrodynamic velocities 
$v_{l, \mathbf{k}}(a ; t), l=n, j, \varepsilon$ are the linear function of $a$. Another approach for the calculation of hydrodynamical velocities $v_{l, \mathbf{k}}(a ; t)$ was proposed on the basis of local thermodynamics [45]. The resulting expressions are obviously valid at low frequencies and for small values of the wave vector, when the conditions of the local thermodynamics are valid. Structure function $W(a ; t)$ and hydrodynamical velocities $v_{l, \mathbf{k}}(a ; t)$ in a case of study of hydrodynamic fluctuations were calculated in [47, 48] using the method of collective variables [49]. The basic idea of this approach is that the structure function $W(a ; t)$ and hydrodynamic velocities $v_{l, \mathbf{k}}(a ; t)$ can be calculated in approximations higher than Gaussian. Next, we use the method of collective variables [35, 47-49] to calculate the structure function $W(a ; t)$ and hydrodynamic velocities $v_{l, \mathbf{k}}(a ; t)$. Further, the collective variable method is used to calculate the structure function $W(a ; t)$ and hydrodynamic velocities $v_{l, \mathbf{k}}(a ; t)$. We considered earlier the case when the interactions between particles on small distances were described by short-range potential $\Phi^{\mathrm{sh}}\left(\left|\mathbf{r}_{l j}\right|\right)$, in particular, by the potential of hard spheres. At long distances, the interactions between particles are described by the long-range potential $\Phi^{\text {long }}\left(\left|\mathbf{r}_{l j}\right|\right)$. Accordingly, we define the short- and long-acting parts of the interaction of the Liouville operator:

$$
\mathrm{i} L_{N}=\mathrm{i} L_{N}^{0}+\hat{T}_{N}+\mathrm{i} L_{N}^{\text {long }}
$$

where $\mathrm{i} L_{N}^{0}$ is the Liouville operator of $N$ non-interacting particles, $\hat{T}_{N}$ is the scattering operator of hard sphere system and $\mathrm{i} L_{N}^{\text {long }}$ is the potential part of Liouville operator with long-range interaction between particles.

First, we calculate the structure function $W(a ; t)$ for collective variables. To do this, we use the integral representation for $\delta$-functions:

$$
\hat{f}(a)=\int \mathrm{d} \omega \exp \left[-\mathrm{i} \pi \sum_{l, \mathbf{k}} \omega_{l, \mathbf{k}}\left(\hat{a}_{l, \mathbf{k}}-a_{l, \mathbf{k}}\right)\right], \quad l=n, \mathbf{j}, \varepsilon .
$$

Next, using a cumulant expansion [35, 48] for $W(a ; t)$, one obtains:

$$
\begin{aligned}
W(a ; t)= & \int \mathrm{d} \Gamma_{N} \varrho_{\mathrm{rel}}^{\mathrm{kin}-\mathrm{hyd}}\left(x^{N} ; t\right) \hat{f}(a)=\int \mathrm{d} \omega \exp \left[-\mathrm{i} \pi \sum_{l, \mathbf{k}} \omega_{l, \mathbf{k}} \bar{a}_{l, \mathbf{k}}-\frac{1}{2 V^{2}} \sum_{\mathbf{q}} \sum_{\mathbf{k}} \beta_{-\mathbf{q}}(t) v(\mathbf{k})\left(n_{\mathbf{q}+\mathbf{k}} n_{-\mathbf{k}}-n_{\mathbf{q}}\right)\right. \\
& \left.-\frac{\pi^{2}}{2} \sum_{l_{1}, l_{2}} \sum_{\mathbf{k}_{1}, \mathbf{k}_{2}} \mathfrak{M}_{2}^{l_{1}, l_{2}}\left(\mathbf{k}_{1}, \mathbf{k}_{2} ; t\right) \omega_{l_{1}, \mathbf{k}_{1}} \omega_{l_{2}, \mathbf{k}_{2}}\right] \exp \left[\sum_{n \geqslant 3} D_{n}(\omega ; t)\right]
\end{aligned}
$$

where

$$
\begin{gathered}
\bar{a}_{l, \mathbf{k}}=a_{l, \mathbf{k}}-\left\langle\hat{a}_{l, \mathbf{k}}\right\rangle_{\text {kin-sh }}^{t}, \quad \mathrm{~d} \omega=\prod_{l, \mathbf{k}} \mathrm{d} \omega_{l, \mathbf{k}}^{r} \mathrm{~d} \omega_{l, \mathbf{k}}^{s}, \quad \omega_{l, \mathbf{k}}=\omega_{l, \mathbf{k}}^{r}-\mathrm{i} \omega_{l, \mathbf{k}}^{s}, \quad \omega_{l,-\mathbf{k}}=\omega_{l, \mathbf{k}}^{*}, \\
D_{n}(\omega ; t)=\frac{(-\mathrm{i} \pi)^{n}}{n !} \sum_{l_{1}, \ldots, l_{n} \mathbf{k}_{1}, \ldots, \mathbf{k}_{n}} \sum_{n} \mathfrak{M}_{n}^{l_{1}, \ldots, l_{n}}\left(\mathbf{k}_{1}, \ldots, \mathbf{k}_{n} ; t\right) \omega_{l_{1}, \mathbf{k}_{1}} \ldots \omega_{l_{n}, \mathbf{k}_{n}}, \\
\mathfrak{M}_{n}^{l_{1}, \ldots, l_{n}}\left(\mathbf{k}_{1}, \ldots, \mathbf{k}_{n} ; t\right)=\left\langle\hat{a}_{l_{1}, \mathbf{k}_{1}} \ldots \hat{a}_{l_{n}, \mathbf{k}_{n}}\right\rangle_{\text {kin-sh }}^{t, \mathrm{c}}
\end{gathered}
$$

are the non-equilibrium cumulant averages in approximations of the $n$-order, which are calculated using distribution $\varrho_{\text {rel }}^{\mathrm{kin}-\mathrm{sh}}\left(x^{N} ; t\right)$ for hard sphere model:

$$
\varrho_{\text {rel }}^{\text {kin-sh }}\left(x^{N} ; t\right)=\exp \left[-\Phi^{\text {kin-sh }}(t)-\int \mathrm{d} \mathbf{r} \beta(\mathbf{r} ; t) \hat{H}^{\mathrm{sh}}(\mathbf{r})-\int \mathrm{d} x \gamma(x ; t) \hat{n}_{1}(x)\right] .
$$

In (3.4) superscript "c" means the cumulant averages. It should be noted that in 3.2 we shared the contributions from short- and long-range interactions. The short-range interactions are take into account in a relevant distribution (3.5) (which can be considered as a base distribution) and long-range interactions are presented through collective variables

$$
\int \mathrm{d} \mathbf{r} \beta(\mathbf{r} ; t) \hat{H}^{\text {long }}(\mathbf{r})=\frac{1}{2 V^{2}} \sum_{\mathbf{q}} \sum_{\mathbf{k}} \beta_{-\mathbf{q}}(t) v(\mathbf{k})\left(n_{\mathbf{q}+\mathbf{k}} n_{-\mathbf{k}}-n_{\mathbf{q}}\right) .
$$


We present the structure function $W(a ; t)$ for further calculations in the following form:

$$
\begin{aligned}
W(a ; t)= & \int \mathrm{d} \omega \exp \left[-\mathrm{i} \pi \sum_{l, \mathbf{k}} \omega_{l, \mathbf{k}} \bar{a}_{l, \mathbf{k}}-\frac{1}{2 V^{2}} \sum_{\mathbf{q}} \sum_{\mathbf{k}} \beta_{-\mathbf{q}}(t) v(\mathbf{k})\left(n_{\mathbf{q}+\mathbf{k}} n_{-\mathbf{k}}-n_{\mathbf{q}}\right)\right. \\
& \left.-\frac{\pi^{2}}{2} \sum_{l_{1}, l_{2}} \sum_{\mathbf{k}_{1}, \mathbf{k}_{2}} \mathfrak{M}_{2}^{l_{1}, l_{2}}\left(\mathbf{k}_{1}, \mathbf{k}_{2} ; t\right) \omega_{l_{1}, \mathbf{k}_{1}} \omega_{l_{2}, \mathbf{k}_{2}}\right]\left(1+B+\frac{1}{2 !} B^{2}+\frac{1}{3 !} B^{3}+\ldots+\frac{1}{n !} B^{n}+\ldots\right),
\end{aligned}
$$

where $B=\sum_{n \geqslant 3} D_{n}(\omega ; t)$. If in series of exponent (3.6), namely, $\exp \left[\sum_{n \geqslant 3} D_{n}(\omega ; t)\right]$, one retains only the first term equal to unity, one will obtain the Gaussian approximation for $W(a ; t)$ :

$$
\begin{aligned}
W^{\mathrm{G}}(a ; t)= & \int \mathrm{d} \omega \exp \left[\mathrm{i} \pi \sum_{l, \mathbf{k}} \omega_{l, \mathbf{k}} \bar{a}_{l, \mathbf{k}}-\frac{1}{2 V^{2}} \sum_{\mathbf{q}} \sum_{\mathbf{k}} \beta_{-\mathbf{q}}(t) v(\mathbf{k})\left(n_{\mathbf{q}+\mathbf{k}} n_{-\mathbf{k}}-n_{\mathbf{q}}\right)\right. \\
& \left.-\frac{\pi^{2}}{2} \sum_{l_{1}, l_{2}} \sum_{\mathbf{k}_{1}, \mathbf{k}_{2}} \mathfrak{M}_{2}^{l_{1}, l_{2}}\left(\mathbf{k}_{1}, \mathbf{k}_{2} ; t\right) \omega_{l_{1}, \mathbf{k}_{1}} \omega_{l_{2}, \mathbf{k}_{2}}\right],
\end{aligned}
$$

where $\mathfrak{M}_{2}^{l_{1}, l_{2}}\left(\mathbf{k}_{1}, \mathbf{k}_{2} ; t\right)$ are the matrix elements of non-equilibrium correlation functions:

$$
\mathfrak{M}_{2}\left(\mathbf{k}_{1}, \mathbf{k}_{2} ; t\right)=\left|\begin{array}{ccc}
\langle\hat{n} \hat{n}\rangle_{\text {kin-sh }}^{\mathrm{c}} & \langle\hat{n} \hat{\mathbf{J}}\rangle_{\text {kin-sh }}^{\mathrm{c}} & \langle\hat{n} \hat{\varepsilon}\rangle_{\text {kin-sh }}^{\mathrm{c}} \\
\langle\hat{\mathbf{J}} \hat{n}\rangle_{\text {kin-sh }}^{\mathrm{c}} & \langle\hat{\mathbf{J}} \hat{\jmath}\rangle_{\text {kin-sh }}^{\mathrm{c}} & \langle\hat{\mathbf{J}} \hat{\varepsilon}\rangle_{\text {kin-sh }}^{\mathrm{c}} \\
\langle\hat{\varepsilon} \hat{n}\rangle_{\text {kin-sh }}^{\mathrm{c}} & \langle\hat{\varepsilon} \hat{\mathbf{J}}\rangle_{\text {kin-sh }}^{\mathrm{c}} & \langle\hat{\varepsilon} \hat{\varepsilon}\rangle_{\text {kin-sh }}^{\mathrm{c}}
\end{array}\right|_{\mathbf{k}_{1}, \mathbf{k}_{2}},
$$

and, for example, the non-equilibrium cumulant average

$$
\left\langle\hat{n}_{\mathbf{k}} \hat{n}_{-\mathbf{k}}\right\rangle_{\mathrm{kin}-\mathrm{sh}}^{t, \mathrm{c}}=\left\langle\hat{n}_{\mathbf{k}} \hat{n}_{-\mathbf{k}}\right\rangle_{\mathrm{kin}-\mathrm{sh}}^{t}-\left\langle\hat{n}_{\mathbf{k}}\right\rangle_{\mathrm{kin}-\mathrm{sh}}^{t}\left\langle\hat{n}_{-\mathbf{k}}\right\rangle_{\mathrm{kin}-\mathrm{sh}}^{t} \cdot
$$

For integrating over $\mathrm{d} \omega$ in (3.7), we should transform the quadratic form in an exponential expression into a diagonal form with respect to $\omega_{l, \mathbf{k}}$. To this end, it is necessary to find the eigenvalues of the matrix (3.8) by solving the equation

$$
\operatorname{det}\left|\tilde{\mathfrak{M}}_{2}\left(\mathbf{k}_{1}, \mathbf{k}_{2} ; t\right)-\tilde{E}(\mathbf{k} ; t)\right|=0,
$$

$\tilde{E}(\mathbf{k} ; t)$ is the diagonal matrix. Further, the expression 3.7 can be written as follows:

$$
\begin{aligned}
W^{\mathrm{G}}(a ; t)= & \int \mathrm{d} \tilde{\omega} \operatorname{det} \tilde{W} \exp \left[-\mathrm{i} \pi \sum_{l, \mathbf{k}} \tilde{a}_{l \mathbf{k}} \tilde{\omega}_{l \mathbf{k}}-\frac{1}{2 V^{2}} \sum_{\mathbf{q}} \sum_{\mathbf{k}} \beta_{-\mathbf{q}}(t) v(\mathbf{k})\left(n_{\mathbf{q}+\mathbf{k}} n_{-\mathbf{k}}-n_{\mathbf{q}}\right)\right. \\
& \left.-\frac{\pi^{2}}{2} \sum_{l} \sum_{\mathbf{k}} E_{l}(\mathbf{k} ; t) \tilde{\omega}_{l \mathbf{k}} \tilde{\omega}_{l,-\mathbf{k}}\right],
\end{aligned}
$$

where new variables $\tilde{a}_{l \mathbf{k}}, \tilde{\omega}_{l \mathbf{k}}$ are connected with the old variables by the ratio:

$$
\tilde{a}_{n \mathbf{k}}=\sum_{l} \bar{a}_{l \mathbf{k}} \omega_{l n}, \quad \omega_{l \mathbf{k}}=\sum_{m=1}^{3} \omega_{l m} \tilde{\omega}_{m \mathbf{k}},
$$

and $\omega_{l m}$ are matrix elements:

$$
\tilde{W}=\left|\begin{array}{ccc}
\omega_{11} & \cdots & \omega_{15} \\
\vdots & \ddots & \vdots \\
\omega_{51} & \cdots & \omega_{55}
\end{array}\right|_{(\mathbf{k} ; t)}
$$

Integrand in 3.11) is a quadratic function $\tilde{\omega}_{n \mathbf{k}}$ and after integrating over $\mathrm{d} \omega_{n \mathbf{k}}$ we will obtain the following structure function in Gaussian approximation $W^{\mathrm{G}}(a ; t)$ :

$$
\begin{aligned}
W^{\mathrm{G}}(a ; t)= & \exp \left[-\frac{1}{2} \sum_{l, \mathbf{k}} E_{l}^{-1}(\mathbf{k} ; t) \tilde{a}_{l \mathbf{k}} \tilde{a}_{l,-\mathbf{k}}-\frac{1}{2 V^{2}} \sum_{\mathbf{q}} \sum_{\mathbf{k}} \beta_{-\mathbf{q}}(t) v(\mathbf{k})\left(n_{\mathbf{q}+\mathbf{k}} n_{-\mathbf{k}}-n_{\mathbf{q}}\right)\right] \\
& \times \exp \left[-\frac{1}{2} \sum_{\mathbf{k}} \ln \pi \operatorname{det} \tilde{E}(\mathbf{k} ; t)\right] \exp \left[\sum_{\mathbf{k}} \ln \operatorname{det} \tilde{W}(\mathbf{k} ; t)\right],
\end{aligned}
$$


or through variables $\bar{a}_{l \mathbf{k}}$ :

$$
W^{\mathrm{G}}(a ; t)=Z(t) \exp \left[-\frac{1}{2} \sum_{l, \mathbf{k}} \bar{E}_{l}(\mathbf{k} ; t) \bar{a}_{l \mathbf{k}} \bar{a}_{l,-\mathbf{k}}-\frac{1}{2 V^{2}} \sum_{\mathbf{q}} \sum_{\mathbf{k}} \beta_{-\mathbf{q}}(t) v(\mathbf{k})\left(n_{\mathbf{q}+\mathbf{k}} n_{-\mathbf{k}}-n_{\mathbf{q}}\right)\right],
$$

where

$$
\begin{gathered}
\bar{E}_{l}(\mathbf{k} ; t)=\sum_{l^{\prime}} \omega_{l l^{\prime}} E_{l^{\prime}}^{-1}(\mathbf{k} ; t) \omega_{l^{\prime} l}, \\
Z(t)=\exp \left[-\frac{1}{2} \sum_{\mathbf{k}} \ln \pi \operatorname{det} \tilde{E}(\mathbf{k} ; t)\right] \exp \left[\sum_{\mathbf{k}} \ln \operatorname{det} \tilde{W}(\mathbf{k} ; t)\right] .
\end{gathered}
$$

The structure function $W^{\mathrm{G}}(a ; t)$ provides a possibility to calculate (3.2) in higher approximations over Gaussian moments [35, 48]:

$$
W(a ; t)=W^{\mathrm{G}}(a ; t) \exp \left[\sum_{n \geqslant 3}\left\langle\tilde{D}_{n}(a ; t)\right\rangle_{\mathrm{G}}\right]
$$

where one presents $\left\langle\tilde{D}_{n}(a ; t)\right\rangle_{\mathrm{G}}$ approximately as:

$$
\begin{gathered}
\left\langle\tilde{D}_{3}(a ; t)\right\rangle_{\mathrm{G}}=\left\langle\bar{D}_{3}(a ; t)\right\rangle_{\mathrm{G}}, \\
\left\langle\tilde{D}_{4}(a ; t)\right\rangle_{\mathrm{G}}=\left\langle\bar{D}_{4}(a ; t)\right\rangle_{\mathrm{G}}, \\
\left\langle\tilde{D}_{6}(a ; t)\right\rangle_{\mathrm{G}}=\left\langle\bar{D}_{6}(a ; t)\right\rangle_{\mathrm{G}}-\frac{1}{2}\left\langle\bar{D}_{3}(a ; t)\right\rangle_{\mathrm{G}}^{2}, \\
\left\langle\tilde{D}_{8}(a ; t)\right\rangle_{\mathrm{G}}=\left\langle\bar{D}_{8}(a ; t)\right\rangle_{\mathrm{G}}-\left\langle\bar{D}_{3}(a ; t)\right\rangle_{\mathrm{G}}\left\langle\bar{D}_{5}(a ; t)\right\rangle_{\mathrm{G}}-\frac{1}{2}\left\langle\bar{D}_{4}(a ; t)\right\rangle_{\mathrm{G}}^{2}, \\
\left\langle\tilde{D}_{n}(a ; t)\right\rangle_{\mathrm{G}}=\frac{1}{W^{\mathrm{G}}(a ; t)} \sum_{l_{1}, \ldots, l_{n} \mathbf{k}_{1}, \ldots, \mathbf{k}_{n}} \overline{\mathfrak{M}}_{n}^{l_{1}, \ldots, l_{n}}\left(\mathbf{k}_{1}, \ldots, \mathbf{k}_{n} ; t\right) \frac{1}{(\mathrm{i} \pi)^{n}} \frac{\delta^{n}}{\delta \bar{a}_{l_{1}, \mathbf{k}_{1}} \ldots \delta \bar{a}_{l_{n}, \mathbf{k}_{n}}} W^{\mathrm{G}}(a ; t) .
\end{gathered}
$$

$\left\langle\tilde{D}_{n}(a ; t)\right\rangle_{\mathrm{G}}$ are the renormalized non-equilibrium cumulant averages of the order $n$ for the variables $\bar{a}_{l \mathbf{k}}$. In expression (3.14), the summands are with only even degrees over $a$ since all odd Gaussian moments vanish.

The method of calculation of the structure function $W(a ; t)$ can be used for approximate calculations of hydrodynamic velocities $v_{l, \mathbf{k}}(a ; t)$. We present a general formula of velocities consistent with (2.30) in the following form:

$$
v_{l, \mathbf{k}}(a ; t)=\int \mathrm{d} \Gamma_{N} \dot{\hat{a}}_{l, \mathbf{k}} \varrho_{\mathrm{rel}}^{\text {kin-hyd }}\left(x^{N} ; t\right) \hat{f}(a)
$$

and introduce the function $W(a, \lambda ; t)$ :

$$
W(a, \lambda ; t)=\int \mathrm{d} \Gamma_{N} \exp \left(-\mathrm{i} \pi \sum_{l, \mathbf{k}} \lambda_{l, \mathbf{k}} \dot{\hat{a}}_{l, \mathbf{k}}\right) \varrho_{\text {rel }}^{\text {kin-hyd }}\left(x^{N} ; t\right) \hat{f}(a)
$$

so that

$$
\nu_{l, \mathbf{k}}(a ; t)=\left.\frac{\partial}{\partial\left(-\mathbf{i} \pi \lambda_{l, \mathbf{k}}\right)} \ln W(a, \lambda ; t)\right|_{\lambda_{l, \mathbf{k}}=0} .
$$

We calculate the function $W(a, \lambda ; t)$ using the preliminary results of the calculation of the structural function $W(a ; t)$, and rewrite $W(a, \lambda ; t)$ as:

$$
W(a, \lambda ; t)=\int \mathrm{d} \Gamma_{N} \int \mathrm{d} \omega \exp \left(-\mathrm{i} \pi \sum_{l, \mathbf{k}} \lambda_{l, \mathbf{k}} \dot{\hat{a}}_{l, \mathbf{k}}\right) \exp \left[-\mathrm{i} \pi \sum_{l, \mathbf{k}} \omega_{l, \mathbf{k}}\left(\hat{a}_{l, \mathbf{k}}-a_{l, \mathbf{k}}\right)\right] \varrho_{\text {rel }}^{\text {kin-hyd }}\left(x^{N} ; t\right)
$$

Now, we carry out an averaging in (3.18) using the following cumulant expansion:

$$
\begin{aligned}
W(a, \lambda ; t)= & \int \mathrm{d} \omega \exp \left\{-\mathrm{i} \pi \sum_{l, \mathbf{k}} \omega_{l, \mathbf{k}} \bar{a}_{l, \mathbf{k}}-\frac{1}{2 V^{2}} \sum_{\mathbf{q}} \sum_{\mathbf{k}} \beta_{-\mathbf{q}}(t) v(\mathbf{k})\left(n_{\mathbf{q}+\mathbf{k}} n_{-\mathbf{k}}-n_{\mathbf{q}}\right)\right. \\
& \left.+\sum_{n \geqslant 1}\left[D_{n}(\omega ; t)+D_{n}(\lambda ; t)+D_{n}(\omega, \lambda ; t)\right]\right\},
\end{aligned}
$$


where

$$
\begin{aligned}
D_{n}(\omega ; t) & =\frac{(-\mathrm{i} \pi)^{n}}{n !} \sum_{l_{1}, \ldots, l_{n} \mathbf{k}_{1}, \ldots, \mathbf{k}_{n}} \mathfrak{M}_{n}^{l_{1}, \ldots, l_{n}}\left(\mathbf{k}_{1}, \ldots, \mathbf{k}_{n} ; t\right) \omega_{l_{1}, \mathbf{k}_{1}} \ldots \omega_{l_{n}, \mathbf{k}_{n}}, \\
D_{n}(\lambda ; t) & =\frac{(-\mathrm{i} \pi)^{n}}{n !} \sum_{l_{1}, \ldots, l_{n} \mathbf{k}_{1}, \ldots, \mathbf{k}_{n}} \mathfrak{M}_{n}^{(1) l_{1}, \ldots, l_{n}}\left(\mathbf{k}_{1}, \ldots, \mathbf{k}_{n} ; t\right) \lambda_{l_{1}, \mathbf{k}_{1} \ldots \lambda_{l_{n}, \mathbf{k}_{n}},} \\
D_{n}(\omega, \lambda ; t) & =\frac{(-\mathrm{i} \pi)^{n}}{n !} \sum_{l_{1}, \ldots, l_{n} \mathbf{k}_{1}, \ldots, \mathbf{k}_{n}} \sum \mathfrak{M}_{n}^{(2) l_{1}, \ldots, l_{n}}\left(\mathbf{k}_{1}, \ldots, \mathbf{k}_{n} ; t\right) \omega_{l_{1}, \mathbf{k}_{1}} \ldots \omega_{l_{n-1}, \mathbf{k}_{n-1}} \ldots \lambda_{l_{n}, \mathbf{k}_{n}},
\end{aligned}
$$

with the cumulants of the following structure:

$$
\begin{aligned}
& \mathfrak{M}_{n}^{l_{1}, \ldots, l_{n}}\left(\mathbf{k}_{1}, \ldots, \mathbf{k}_{n} ; t\right)=\left\langle\hat{a}_{l_{1}, \mathbf{k}_{1}}, \ldots, \hat{a}_{l_{n}, \mathbf{k}_{n}}\right\rangle_{\text {kin-sh }}^{t, \mathrm{c}}, \\
& \mathfrak{M}_{n}^{(1) l_{1}, \ldots, l_{n}}\left(\mathbf{k}_{1}, \ldots, \mathbf{k}_{n} ; t\right)=\left\langle\dot{\hat{a}}_{l_{1}, \mathbf{k}_{1}}, \ldots, \dot{\hat{a}}_{l_{n}, \mathbf{k}_{n}}\right\rangle_{\text {kin-sh }}^{t, \mathrm{c}} \\
& \mathfrak{M}_{n}^{(2) l_{1}, \ldots, l_{n}}\left(\mathbf{k}_{1}, \ldots, \mathbf{k}_{n} ; t\right)=n\left[(n-j)+(j-n+1) \delta_{l_{1}, \ldots, l_{n-1}}\right] \\
& \times\left\langle\hat{a}_{l_{1}, \mathbf{k}_{1}}, \ldots, \hat{a}_{l_{n-j}, \mathbf{k}_{n-j}}, \ldots, \dot{\hat{a}}_{l_{n-j+1}, \mathbf{k}_{n-j+1}}, \ldots, \dot{\hat{a}}_{l_{n}, \mathbf{k}_{n}}\right\rangle_{\mathrm{kin}-\mathrm{sh}}^{t, \mathrm{c}} .
\end{aligned}
$$

First, we consider a Gaussian approximation for $W(a, \lambda ; t)$, namely in the exponent of an integrand we leave only the summands with $n=2$ and linear over $\lambda_{l, \mathbf{k}}$ :

$$
\begin{aligned}
W^{\mathrm{G}}(a, \lambda ; t)= & \int \mathrm{d} \omega \exp \left[\mathrm{i} \pi \sum_{l, \mathbf{k}} \omega_{l, \mathbf{k}} \bar{a}_{l, \mathbf{k}}-\mathrm{i} \pi \sum_{l, \mathbf{k}}\left\langle\dot{\hat{a}}_{l, \mathbf{k}}\right\rangle_{\text {kin-hyd }}^{t, \mathrm{c}} \lambda_{l, \mathbf{k}}-\frac{1}{2 V^{2}} \sum_{\mathbf{q}} \sum_{\mathbf{k}} \beta_{-\mathbf{q}}(t) v(\mathbf{k})\left(n_{\mathbf{q}+\mathbf{k}} n_{-\mathbf{k}}-n_{\mathbf{q}}\right)\right. \\
& \left.-\frac{\pi^{2}}{2} \sum_{l_{1}, l_{2}} \sum_{\mathbf{k}_{1}, \mathbf{k}_{2}} \mathfrak{M}_{2}^{l_{1}, l_{2}}\left(\mathbf{k}_{1}, \mathbf{k}_{2} ; t\right) \omega_{l_{1}, \mathbf{k}_{1}} \omega_{l_{2}, \mathbf{k}_{2}}-\frac{\pi^{2}}{2} \sum_{l_{1}, l_{2}} \sum_{\mathbf{k}_{1}, \mathbf{k}_{2}} \mathfrak{M}_{2}^{(2) l_{1}, l_{2}}\left(\mathbf{k}_{1}, \mathbf{k}_{2} ; t\right) \omega_{l_{1}, \mathbf{k}_{1}} \lambda_{l_{2}, \mathbf{k}_{2}}\right] .
\end{aligned}
$$

Then, transforming this expression in the exponent to a diagonal quadratic form over variables $\omega_{l, \mathbf{k}}$, similarly to $W(a ; t)$, after integrating with respect to the new variables $\bar{\omega}_{l, \mathbf{k}}$, one obtains:

$$
\begin{aligned}
W^{\mathrm{G}}(a, \lambda ; t)= & \int \mathrm{d} \omega \exp \left[-\mathrm{i} \pi \sum_{l, \mathbf{k}}\left\langle\dot{\hat{a}}_{l, \mathbf{k}}\right\rangle_{\mathrm{kin}}^{t} \lambda_{l, \mathbf{k}}-\frac{1}{2 V^{2}} \sum_{\mathbf{q}} \sum_{\mathbf{k}} \beta_{-\mathbf{q}}(t) v(\mathbf{k})\left(n_{\mathbf{q}+\mathbf{k}} n_{-\mathbf{k}}-n_{\mathbf{q}}\right)\right. \\
& \left.-\frac{\pi^{2}}{2} \sum_{l, \mathbf{k}} E_{l}^{-1}(\mathbf{k} ; t) b_{l, \mathbf{k}} b_{l,-\mathbf{k}}-\frac{1}{2} \sum_{\mathbf{k}} \ln \pi \operatorname{det} \tilde{E}(\mathbf{k} ; t)+\sum_{\mathbf{k}} \ln \operatorname{det} \tilde{W}(\mathbf{k} ; t)\right]
\end{aligned}
$$

where

$$
b_{l, \mathbf{k}}=\sum_{j} \omega_{l j}\left[\bar{a}_{j, \mathbf{k}}+\frac{\mathrm{i} \pi}{2} \sum_{j^{\prime}} \mathfrak{M}_{2}^{(2) j, j^{\prime}}(\mathbf{k} ; t) \lambda_{j^{\prime}, \mathbf{k}}\right],
$$

and $\omega_{l j}, \mathfrak{M}_{2}^{(2) j, j^{\prime}}(\mathbf{k} ; t)$ and $E_{l}(\mathbf{k} ; t)$ are not dependent on $\lambda_{l, \mathbf{k}}$. Here, the cumulants $\mathfrak{M}_{2}^{(2) j, j^{\prime}}(\mathbf{k} ; t)$ have the following structure:

$$
\mathfrak{M}_{2}^{(2) j, j^{\prime}}(\mathbf{k} ; t)=\left\langle\dot{\hat{a}}_{j, \mathbf{k}} \hat{a}_{j^{\prime},-\mathbf{k}}\right\rangle_{\text {kin-sh }}^{t}-\left\langle\dot{\hat{a}}_{j, \mathbf{k}}\right\rangle_{\text {kin }}^{t}\left\langle\hat{a}_{j^{\prime},-\mathbf{k}}\right\rangle_{\text {kin-sh }}^{t} \cdot
$$

Now, we calculate the hydrodynamic velocities $v_{l, \mathbf{k}}(a ; t)$ in a Gaussian approximation according to the formula

$$
v_{l, \mathbf{k}}(a ; t)=\left.\frac{\partial}{\partial\left(-\mathrm{i} \pi \lambda_{l, \mathbf{k}}\right)} \ln W^{\mathrm{G}}(a, \lambda ; t)\right|_{\lambda_{l, \mathbf{k}}=0}=\left\langle\dot{\hat{a}}_{j, \mathbf{k}}\right\rangle_{\mathrm{kin}}^{t}-\frac{1}{2} \sum_{j, j^{\prime}} E_{l}^{-1}(\mathbf{k} ; t) \omega_{j l} \omega_{j^{\prime} l} \mathfrak{M}_{2}^{(2) j^{\prime}, l}(\mathbf{k} ; t) \bar{a}_{l, \mathbf{k}} .
$$

Specifically, we consider the particular case when one can divide the longitudinal and transverse fluctuations for collective variables. That is, we choose the direction of the wave vector $\mathbf{k}$ along the axis of $\mathrm{Oz}$. Thus, one obtains:

$$
\begin{aligned}
W^{\mathrm{G}}(a ; t)= & \int \mathrm{d} \omega \exp \left[\mathrm{i} \pi \sum_{l, \mathbf{k}} \omega_{l, \mathbf{k}} \bar{a}_{l, \mathbf{k}}-\frac{1}{2 V^{2}} \sum_{\mathbf{q}} \sum_{\mathbf{k}} \beta_{-\mathbf{q}}(t) v(\mathbf{k})\left(n_{\mathbf{q}+\mathbf{k}} n_{-\mathbf{k}}-n_{\mathbf{q}}\right)\right. \\
& -\frac{\pi^{2}}{2} \sum_{l_{1}, l_{2}=1}^{3} \sum_{\mathbf{k}_{1}, \mathbf{k}_{2}} \mathfrak{M}_{2}^{\|, l_{1}, l_{2}}\left(\mathbf{k}_{1}, \mathbf{k}_{2} ; t\right) \omega_{l_{1}, \mathbf{k}_{1}} \omega_{l_{2}, \mathbf{k}_{2}} \\
& \left.-\frac{\pi^{2}}{2} \sum_{l_{1}, l_{2}=1}^{4} \sum_{\mathbf{k}_{1}, \mathbf{k}_{2}} \mathfrak{M}_{2}^{\|, \perp, l_{1}, l_{2}}\left(\mathbf{k}_{1}, \mathbf{k}_{2} ; t\right) \omega_{l_{1}, \mathbf{k}_{1}} \omega_{l_{2}, \mathbf{k}_{2}}\right]
\end{aligned}
$$


where $\mathfrak{M}_{2}^{\|, l_{1}, l_{2}}\left(\mathbf{k}_{1}, \mathbf{k}_{2} ; t\right)$ are the matrix elements of the non-equilibrium correlation functions of longitudinal fluctuations

$$
\mathfrak{M}_{2}^{\|}\left(\mathbf{k}_{1}, \mathbf{k}_{2} ; t\right)=\left|\begin{array}{ccc}
\langle\hat{n} \hat{n}\rangle_{\text {kin-sh }}^{\mathrm{c}} & \left\langle\hat{n} \hat{\mathbf{J}}^{\|}\right\rangle_{\text {kin-sh }}^{\mathrm{c}} & \langle\hat{n} \hat{\varepsilon}\rangle_{\text {kin-sh }}^{\mathrm{c}} \\
\langle\hat{\mathbf{J}} \| \hat{n}\rangle_{\text {kin-sh }}^{\mathrm{c}} & \left\langle\hat{\mathbf{J}}^{\|} \hat{\mathbf{J}}^{\|}\right\rangle_{\text {kin-sh }}^{\mathrm{c}} & \langle\hat{\mathbf{J}} \| \hat{\varepsilon}\rangle_{\text {kin-sh }}^{\mathrm{c}} \\
\langle\hat{\varepsilon} \hat{n}\rangle_{\text {kin-sh }}^{\mathrm{c}} & \left\langle\hat{\varepsilon} \hat{\mathbf{J}}^{\|}\right\rangle_{\text {kin-sh }}^{\mathrm{c}} & \langle\hat{\varepsilon} \hat{\varepsilon}\rangle_{\text {kin-sh }}^{\mathrm{c}}
\end{array}\right|_{\mathbf{k}_{1}, \mathbf{k}_{2}},
$$

$\mathfrak{M}_{2}^{\perp l_{1}, l_{2}}\left(\mathbf{k}_{1}, \mathbf{k}_{2} ; t\right)$ are the matrix elements of the non-equilibrium correlation functions of transverse and transverse-longitudinal fluctuations

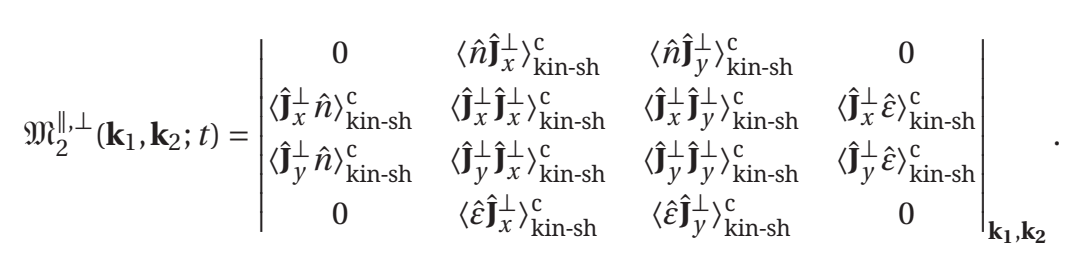

In this case, the hydrodynamic velocities in the Gaussian approximation are as follows:

$$
\begin{aligned}
& v_{n \mathbf{k}}^{\| \mathrm{G}}(a ; t)=\left\langle\dot{\hat{n}}_{\mathbf{k}}\right\rangle_{\text {kin-sh }}^{t}+E_{1}^{-1}(\mathbf{k} ; t)\left(\omega_{11} \bar{n}_{\mathbf{k}}+\omega_{21} \overline{\mathbf{J}}_{\mathbf{k}}^{\|}+\omega_{31} \bar{\varepsilon}_{\mathbf{k}}\right) \Omega_{n}(\mathbf{k} ; t), \\
& v_{J \mathbf{k}}^{\| \mathrm{G}}(a ; t)=\left\langle\dot{\hat{J}}_{\mathbf{k}}^{\|}\right\rangle_{\text {kin-sh }}^{t}+E_{2}^{-1}(\mathbf{k} ; t)\left(\omega_{12} \bar{n}_{\mathbf{k}}+\omega_{22} \overline{\mathbf{J}}_{\mathbf{k}}^{\|}+\omega_{32} \bar{\varepsilon}_{\mathbf{k}}\right) \Omega_{J}(\mathbf{k} ; t), \\
& v_{\varepsilon \mathbf{k}}^{\| G}(a ; t)=\left\langle\dot{\hat{\varepsilon}}_{\mathbf{k}}\right\rangle_{\text {kin-sh }}^{t}+E_{3}^{-1}(\mathbf{k} ; t)\left(\omega_{13} \bar{n}_{\mathbf{k}}+\omega_{23} \bar{J}_{\mathbf{k}}^{\|}+\omega_{33} \bar{\varepsilon}_{\mathbf{k}}\right) \Omega_{\varepsilon}(\mathbf{k} ; t),
\end{aligned}
$$

where

$$
\begin{aligned}
& \Omega_{n}(\mathbf{k} ; t)=\omega_{11}\left\langle\hat{n}_{\mathbf{k}} \dot{\hat{n}}_{-\mathbf{k}}\right\rangle_{\text {kin-sh }}^{t, \mathrm{c}}+\omega_{21}\left\langle\hat{\mathbf{J}}_{\mathbf{k}}^{\|} \dot{\hat{n}}_{-\mathbf{k}}\right\rangle_{\text {kin-sh }}^{t, \mathrm{c}}+\omega_{31}\left\langle\hat{\varepsilon}_{\mathbf{k}} \dot{\hat{\hat{n}}}_{-\mathbf{k}}\right\rangle_{\text {kin-sh }}^{t, \mathrm{c}}, \\
& \Omega_{J}(\mathbf{k} ; t)=\omega_{12}\left\langle\hat{n}_{\mathbf{k}} \dot{\hat{\mathbf{J}}}_{-\mathbf{k}}^{\|}\right\rangle_{\text {kin-sh }}^{t, \mathrm{c}}+\omega_{22}\left\langle\hat{\mathbf{J}}_{\mathbf{k}}^{\| \dot{\hat{J}}_{-\mathbf{k}}^{\|}}\right\rangle_{\text {kin-sh }}^{t, \mathrm{c}}+\omega_{32}\left\langle\hat{\varepsilon}_{\mathbf{k}} \dot{\hat{\mathbf{J}}}_{-\mathbf{k}}^{\|}\right\rangle_{\text {kin-sh}}^{t, \mathrm{c}}, \\
& \Omega_{\varepsilon}(\mathbf{k} ; t)=\omega_{13}\left\langle\hat{n}_{\mathbf{k}} \dot{\hat{\varepsilon}}_{-\mathbf{k}}\right\rangle_{\text {kin-sh }}^{t, \mathrm{c}}+\omega_{23}\left\langle\hat{\mathbf{J}}_{\mathbf{k}}^{\|} \dot{\hat{\varepsilon}}_{-\mathbf{k}}\right\rangle_{\text {kin-sh }}^{t, \mathrm{c}}+\omega_{33}\left\langle\hat{\varepsilon}_{\mathbf{k}} \dot{\hat{\varepsilon}}_{-\mathbf{k}}\right\rangle_{\text {kin-sh}}^{t, \mathrm{c}}
\end{aligned}
$$

and $\omega_{l j}$ are the elements of matrix $\tilde{W}(\mathbf{k} ; t)$.

As one can see the hydrodynamic velocities 3.27) in the Gaussian approximation for $W^{\mathrm{G}}(a, \lambda ; t)$ are linear functions of the collective variables $n_{\mathbf{k}}, \mathbf{J}_{\mathbf{k}}$ and $\varepsilon_{\mathbf{k}}$. It is remarkable that if the kinetic processes are not taken into account, i.e., $\varrho_{\text {rel }}^{\mathrm{kin}-\mathrm{sh}}\left(x^{N} ; t\right)=1$, then $\langle\ldots\rangle_{\mathrm{kin}-\mathrm{sh}}^{t} \rightarrow\langle\ldots\rangle_{0}$ is an average over a microscopic ensemble $W(a)$; in this case, the expressions 3.27 for hydrodynamic velocities transform into the results of the previous work [48], in which the nonlinear hydrodynamic fluctuations in simple fluids were investigated.

\section{Conclusions}

Using the method of Zubarev non-equilibrium statistical operator, we have obtained a modified chain of BBGKY kinetic equations that take into account non-equilibrium hydrodynamic fluctuations for a system of interacting particles. At the same time, the non-equilibrium distribution function that describes the hydrodynamic fluctuations, satisfies a generalized Fokker-Planck equation. We divide the contributions from short-range and long-range interactions between particles, and describe the short-range interactions (hard sphere model) in the coordinate space, while the long-range interactions are described in the space of collective variables.

Moreover, the short-range component will be considered as a basis with distribution $\varrho_{\text {rel }}^{\text {kin-sh }}\left(x^{N} ; t\right)$, which corresponds to the BBGKY chain of equations for the model of hard spheres [40].

The used method of collective variables [35, 47, 49] has made it possible to calculate the structural distribution function of hydrodynamic collective variables and their hydrodynamic velocities in approximations higher than the Gaussian one. In particular, the hydrodynamic velocities above the Gaussian approximation that follow from equation (3.19) and (3.27) will be proportional to $\bar{a}_{l, \mathbf{k}} \bar{a}_{l^{\prime}, \mathbf{k}}$, and the transport kernels in the Fokker-Planck equation will be of fourth-order correlation functions over variables $\hat{a}_{l, \mathbf{k}}$. It is significant that the Fokker-Planck equation in a Gauss approximation for $\tilde{W}^{\mathrm{G}}(\mathbf{k} ; t), v_{l, \mathbf{k}}^{\mathrm{G}}(a ; t)$ leads to 
transport equations for $\left\langle\hat{a}_{l, \mathbf{k}}\right\rangle^{t}$ and the structure of these equations is the same as in molecular hydrodynamics [50, 51], although the averaging is performed using $\varrho_{\mathrm{L}}\left(x^{N}, a ; t\right)=\varrho_{\mathrm{rel}}^{\mathrm{kin}-\mathrm{hyd}}\left(x^{N} ; t\right) \hat{f}(a) / W^{\mathrm{G}}(a ; t)$.

The proposed approach makes it possible to go beyond the Gaussian approximation for $\tilde{W}(\mathbf{k} ; t)$, $v_{l, \mathbf{k}}(a ; t)$ and for transport kernels in the Fokker-Planck equation. As a result, we obtain a nonlinear system of equations for the $\left\langle\hat{a}_{l, \mathbf{k}}\right\rangle^{t}$. It is remarkable that the kinetic equation (2.39) contains generalized integrals of Fokker-Planck type with generalized coefficients of diffusion and friction in the phase space $(\mathbf{r}, \mathbf{p}, t)$, where the limit of change of $|\mathbf{r}|$ is restricted by value $|\mathbf{k}|_{\text {hydr }}^{-1}$ that corresponds to collective nonlinear hydrodynamic fluctuations. This means that in areas smaller than $|\mathbf{k}|_{\text {hydr }}^{-1}$, the processes are described by generalized coefficients of diffusion and friction, while in areas larger than $|\mathbf{k}|_{\text {hydr }}^{-1}$ they are described by generalized coefficient of viscosity, thermal conductivity $\phi_{j j}\left(\mathbf{k}, \mathbf{q}, a, a^{\prime} ; t, t^{\prime}\right), \phi_{\varepsilon \varepsilon}\left(\mathbf{k}, \mathbf{q}, a, a^{\prime} ; t, t^{\prime}\right)$ and by cross coefficients $\phi_{j \varepsilon}\left(\mathbf{k}, \mathbf{q}, a, a^{\prime} ; t, t^{\prime}\right), \phi_{\varepsilon j}\left(\mathbf{k}, \mathbf{q}, a, a^{\prime} ; t, t^{\prime}\right)$. The correlations between these areas of transport are described by kernels $\phi_{n j}\left(x, \mathbf{q}, a, a^{\prime} ; t, t^{\prime}\right), \quad \phi_{n \varepsilon}\left(x, \mathbf{q}, a, a^{\prime} ; t, t^{\prime}\right), \quad \phi_{\varepsilon n}\left(\mathbf{k}, x^{\prime}, a, a^{\prime} ; t, t^{\prime}\right)$, $\phi_{j n}\left(\mathbf{k}, x^{\prime}, a, a^{\prime} ; t, t^{\prime}\right)$, which are present both in kinetic and Fokker-Planck equations, and describe crosscorrelation between kinetic and hydrodynamic processes.

\section{References}

1. Bogolubov N.N., In: Studies in Statistical Mechanics Vol. 1, de Boer J., Uhlenbeck G.E. (Eds.), North-Holland, Amsterdam, 1962, 1.

2. Born M., Green H.S., A General Kinetic Theory of Liquids, Cambridge University Press, Cambridge, 1949.

3. Kirkwood J.K., J. Chem. Phys., 1946, 14, 180; doi 10.1063/1.1724117

4. Kirkwood J.K., J. Chem. Phys., 1947, 15, 72; doi 10.1063/1.1746292

5. Yvon J., La Théorie des Fluids et L'équation D’etat: Actualités Scientificues et Industrielles, Hermann and Cie, Paris, 1935.

6. Zubarev D.N., Dokl. Akad. Nauk S.S.S.R., 1961, 140, 92 (in Russian).

7. Zubarev D.N., Dokl. Akad. Nauk S.S.S.R., 1965, 164, 537 (in Russian).

8. Zubarev D.N., Theor. Math. Phys., 1970, 3, 505; doi 10.1007/BF01046515

9. Zwanzig R., J. Chem. Phys., 1960, 33, 1338; doi 10.1063/1.1731409

10. Zwanzig R., Phys. Rev., 1961, 124, 983; doi 10.1103/PhysRev.124.983

11. Zwanzig R., Physica, 1964, 30, 1109; doi 10.1016/0031-8914(64)90102-8

12. Robertson B., Phys. Rev., 1967, 144, 151; doi 10.1103/PhysRev.144.151.

13. Robertson B., Phys. Rev., 1967, 160, 175; doi 10.1103/PhysRev.160.175

14. Kawasaki K., Gunton J.D., Phys. Rev. A, 1973, 8, 2048; doi 10.1103/PhysRevA.8.2048

15. Peletminskii S.V., Yatsenko A.A., Zh. Eksp. Teor. Fiz., 1967, 53, 1327 (in Russian) [Sov. Phys. JETP, 1968, 26, 773].

16. Zubarev D.N., Kalashnikov V.P., Theor. Math. Phys., 1971, 3, 395; doi 10.1007/BF01031594.

17. Zubarev D.N., Nonequilibrium Statistical Thermodynamics, Plenum Press, New York, 1974.

18. Akhiezer A.I., Peletminsky S.V., Methods in Statistical Physics, Springer-Verlag, Berlin, 1979.

19. Zubarev D.N., Morozov V.G., Rëpke G., Statistical Mechanics of Nonequilibrium Processes Vol. 1, Akademie, Berlin, 1996.

20. Zubarev D.N., Morozov V.G., Rëpke G., Statistical Mechanics of Nonequilibrium Processes Vol. 2, Akademie, Berlin, 1996.

21. Lyapilin I.I., Kalashnikov V.P., Nonequilibrium Statistical Operator and its Application to the Kinetics of Paramagnetic Phenomena in Conducting Crystals, Ural Division of the Russian Academy of Sciences, Ekaterinburg, 2008 (in Russian).

22. Kostrobij P.P., Tokarchuk M.V., Markovych B.M., Ignatyuk V.V., Hnativ B.V., Reaction-Diffusion Processes in Systems “Metal-Gas”, Lviv Polytechnic National University, Lviv, 2009 (in Ukrainian).

23. Khamzin A.A., Nigmatullin R.R., Nonequilibrium Statistical Operator Method and its Application to the Kinetics of Ising's Magnets, Kazan State University, Kazan, 2011 (in Russian).

24. Zubarev D.N., Itogi Nauki Tekh. Ser. Sovrem. Probl. Mat., 1980, 15, 131 (in Russian) [J. Sov. Math., 1981, 16, 1509].

25. Zubarev D.N., Tserkovnikov Yu.A., Proc. Steklov Inst. Math., 1988, 175, 139.

26. Bogolyubov N.N. (Jr.), Shumovskii A.S., Yukalov V.I., Theor. Math. Phys., 1984, 60, 921; doi 10.1007/BF01017895

27. Shumovsky A.S., Yukalov V.I., Sov. J. Part. Nucl., 1985, 16, 1274 (in Russian).

28. Yukalov V.I., Phys. Rep., 1991, 208, 395; doi 10.1016/0370-1573(91)90074-V

29. Fischer E.W., Physica A, 1993, 202, 183; doi 10.1016/0378-4371(93)90416-2

30. Olemskoi A.I., Koplyk I.V., Usp. Fiz. Nauk, 1995, 165, 1005 (in Russian); doi 10.3367/UFNr.0165.199510a.1105 
31. Olemskoi A.I., Theory of Structure Transformations in Non-Equilibrium Condensed Matter, Horizons in World Physics Series Vol. 231, NOVA Science Publishers, New York, 1999.

32. Bakai A.S., Fischer E.W., J. Chem. Phys., 2004, 120, 5235; doi $10.1063 / 1.1648300$

33. Onuki A.I., Phase Transition Dynamics, Cambridge University Press, Cambridge, 2004.

34. Das S., Statistical Physics of Liquids at Freezing and Beyond, Cambridge University Press, Cambridge, 2011.

35. Hlushak P.A., Tokarchuk M.V., Physica A, 2016, 443, 231; doi 10.1016/j.physa.2015.09.059

36. Klimontovich Yu.L., Theor. Math. Phys., 1998, 115, 707; doi 10.1007/BF02575494

37. Zubarev D.N., Morozov V.G., Theor. Math. Phys., 1984, 60, 814; doi 10.1007/BF01018982

38. Zubarev D.N., Morozov V.G., Omelyan I.P., Tokarchuk I.P., Theor. Math. Phys., 1991, 87, 412; doi 10.1007/BF01016582

39. Zubarev D.N., Morozov V.G., Omelyan I.P., Tokarchuk I.P., Theor. Math. Phys., 1993, 96, 997; doi 10.1007/BF01019063

40. Kobryn A.E., Omelyan I.P., Tokarchuk M.V., J. Stat. Phys., 1998, 92, 973; doi 10.1023/A:1023044610690

41. Zubarev D.N., Morozov V.G., Proc. Steklov Inst. Math., 1992, 191, 155.

42. Tokarchuk M.V., Omelyan I.P., Kobryn A.E., Condens. Matter Phys., 1998, 1, No. 4(16), 687; doi 10.5488/CMP.1.4.687

43. Markiv B., Omelyan I., Tokarchuk M., J. Stat. Phys., 2014, 155, 843; doi 10.1007/s10955-014-0980-4

44. Cohen E.G.D., Physica, 1962, 28, 1045; doi 10.1016/0031-8914(62)90009-5

45. Zubarev D.N., Morozov D.N., Physica A, 1983, 120, 411; doi 10.1016/0378-4371(83)90062-6

46. Kawasaki K., In: Phase Transition and Critical Phenomena Vol. 5A, Domb C., Green M.S. (Eds.), Academic, New York, 1976, 165-411.

47. Zubarev D.N., Theor. Math. Phys., 1982, 53, 1004; doi 10.1007/BF01014797

48. Idzyk I.M., Ighatyuk V.V., Tokarchuk M.V., Ukr. J. Phys., 1996, 41, No. 10, 1017 (in Ukrainian).

49. Yukhnovskii I.R., Holovko M.F., Statistical Theory of Classical Equilibrium Systems, Naukova Dumka, Kiev, 1980 (in Russian).

50. Mryglod I.M., Tokarchuk M.V., Voprosy Atomnoi Nauki i Tekhniki, 1992, 3, No. 24, 134.

51. Mryglod I.M., Omelyan I.P., Tokarchuk M.V., Mol. Phys., 1995, 84, 235; doi 10.1080/00268979500100181

\title{
Ланцюжок кінетичних рівнянь ББГКІ, метод нерівноважного статистичного оператора та метод колективних змінних в нерівноважній статистичній теорії рідин
}

\author{
I.Р. Юхновський, П.А. Глушак, М.В. Токарчук \\ Інститут фізики конденсованих систем НАН України, вул. Свєнціцького, 1, 79011 Львів, Україна
}

\begin{abstract}
Запропоновано ланцюжок кінетичних рівнянь для нерівноважних одночастинкової, двочастинкової і $s$ частинкової функцій розподілу частинок з урахуванням нелінійних гідродинамічних флуктуацій. Використовується метод нерівноважного статистичного оператора Зубарєва з проектуванням. Нелінійні гідродинамічні флуктуації описуються нерівноважною функцією розподілу колективних змінних, що задовольняє узагальнене рівняння Фоккера-Планка. На основі методу колективних змінних запропоновано спосіб розрахунку нерівноважної структурної функції розподілу колективних змінних та їх гідродинамічних швидкостей (вище гаусового наближення), що містяться в узагальненому рівнянні Фоккера-Планка для нерівноважної функції розподілу колективних змінних. При цьому розділені вклади від короткодіючих і далекодіючих взаємодій між частинками, що привело до того, що короткодіючі взаємодії (наприклад, модель твердих сфер) описуються в координатному просторі, а далекодіючі - у просторі колективних змінних. Короткодіюча складова розглядається як базисна, якій відповідає ланцюжок рівнянь ББгКІ для моделі твердих сфер.
\end{abstract}

Ключові слова: нелінійні флуктуації, нерівноважний статистичний оператор, функція розподілу, рівняння Фоккера-Планка, проста рідина 